J. Appl. Numer. Optim. 2 (2020), No. 2, pp. 213-233

Available online at http://jano.biemdas.com

https://doi.org/10.23952/jano.2.2020.2.07

\title{
TWO INERTIAL LINESEARCH EXTRAGRADIENT ALGORITHMS FOR THE BILEVEL SPLIT PSEUDOMONOTONE VARIATIONAL INEQUALITY WITH CONSTRAINTS
}

\author{
LU-CHUAN CENG
}

Department of Mathematics, Shanghai Normal University, Shanghai 200234, China

\begin{abstract}
In this paper, two modified inertial linesearch extragradient algorithms are investigated for finding a solution of the bilevel split variational inequality problem with the fixed-point constraint of a quasi-nonexpansive mapping. Two strong convergence theorems of solutions are established without the Lipschitz continuity assumption of pseudomonotone mappings.
\end{abstract}

Keywords. Inertial linesearch extragradient method; Bilevel split variational inequality problem; Fixed point problem; Strong convergence

\section{INTRODUCTION}

Let $\mathscr{H}$ be a real Hilbert space and let $C$ be a closed convex nonempty subset. Let $P_{C}$ be the metric projection from $\mathscr{H}$ onto $C$. The following equalities and inequalities are known

(i) $\left\langle x-y, P_{C} x-P_{C} y\right\rangle \geq\left\|P_{C} x-P_{C} y\right\|^{2}, \forall x, y \in \mathscr{H}$;

(ii) $\left\langle x-P_{C} x, y-P_{C} x\right\rangle \leq 0, \forall x \in \mathscr{H}, y \in C$;

(iii) $\|x-y\|^{2} \geq\left\|x-P_{C} x\right\|^{2}+\left\|y-P_{C} x\right\|^{2}, \forall x \in \mathscr{H}, y \in C$;

(iv) $\|x-y\|^{2}=\|x\|^{2}-\|y\|^{2}-2\langle x-y, y\rangle, \forall x, y \in \mathscr{H}$;

(v) $\|s x+(1-s) y\|^{2}=s\|x\|^{2}+(1-s)\|y\|^{2}-s(1-s)\|x-y\|^{2}, \forall x, y \in \mathscr{H}, s \in[0,1]$.

Let $S: C \rightarrow \mathscr{H}$ be a mapping. Recall $S$ is said to be $L$-Lipschitzian (or $L$-Lipschitz continuous) if there exists a constant $L$ such that $\|S x-S y\| \leq L\|x-y\|, \forall x, y \in C$; monotone if $\langle S x-S y, x-y\rangle \geq 0, \forall x, y \in C$; pseudomonotone if $\langle S x, y-x\rangle \geq 0 \Rightarrow\langle S y, y-x\rangle \geq 0, \forall x, y \in C$; $\beta$-strongly monotone if there exists a constant $\beta>0$ such that $\langle S x-S y, x-y\rangle \geq \beta\|x-y\|^{2}$ $\forall x, y \in C$.

Given a vector sequence $\left\{x^{n}\right\} \subset H$, we use the notation $x^{n} \rightarrow x$ (resp., $x^{n} \rightarrow x$ ) to denote the strong (resp., weak) convergence of $\left\{x^{n}\right\}$ to $x$. Given a mapping $S: C \rightarrow \mathscr{H}$, we denote by $\operatorname{Fix}(S)$ the fixed point set of $S$. Let $\Phi: C \rightarrow \mathscr{H}$ be a single-valued mapping. Consider the classical variational inequality problem (VIP), which consists of finding a vector $x^{*}$ in set $C$ such that $\left\langle\Phi x^{*}, x-x^{*}\right\rangle \geq 0, \forall x \in C$. Its solution set is denoted by $\operatorname{Sol}(C, \Phi)$. Recently, efforts and attention have been given to the effective and implementable iterative algorithms for solving the variational inequality; see, e.g., [10, 17, 19, 20, 22]. One of the most popular methods for solving the variational inequality is the extragradient method, studied by Korpelevich

E-mail address: zenglc@ @ shnu.edu.cn.

Received May 14, 2020; Accepted July 30, 2020.

(C)2020 Journal of Applied and Numerical Optimization 
[14] for saddle point problems. Korpelevich's extragradient method and its variants have been extensively studied recently; see, e.g., [4, 12, 15, 24, 25].

In 2011, Censor, Gibali and Reich [4] modified Korpelevich's extragradient method and first introduced the subgradient extragradient method, in which the second projection onto $C$ is replaced by a projection onto a half-space:

$$
\left\{\begin{array}{l}
x^{0} \in \mathscr{H}, \\
y^{n}=P_{C}\left(x^{n}-\ell F x^{n}\right), \\
C_{n}=\left\{y \in \mathscr{H}:\left\langle x^{n}-\ell F x^{n}-y^{n}, y-y^{n}\right\rangle \leq 0\right\}, \\
x^{n+1}=P_{C_{n}}\left(x^{n}-\ell F y^{n}\right), \quad \forall n \geq 0,
\end{array}\right.
$$

where $F: \mathscr{H} \rightarrow \mathscr{H}$ is monotone on $C, \kappa$-Lipschitzian on $\mathscr{H}, \ell \in\left(0, \frac{1}{\kappa}\right)$ and $\operatorname{Sol}(C, F) \neq \emptyset$. They proved that $\left\{x^{n}\right\}$ converges to a solution $x^{*} \in \operatorname{Sol}(C, F)$ weakly.

Let $C$ and $Q$ be two closed convex nonempty subsets of real Hilbert spaces $\mathscr{H}_{1}$ and $\mathscr{H}_{2}$, respectively. Consider the split variational inequality problem (SVIP), which is formulated as follows:

$$
\text { Find } x^{*} \in C:\left\langle F_{1} x^{*}, x-x^{*}\right\rangle \geq 0, \quad \forall x \in C,
$$

such that

$$
y^{*}=A x^{*} \in Q:\left\langle F_{2} y^{*}, y-y^{*}\right\rangle \geq 0, \quad \forall y \in Q,
$$

where $F_{i}$ is a self-mapping on $\mathscr{H}_{i}$ for $i=1,2$ and $A: \mathscr{H}_{1} \rightarrow \mathscr{H}_{2}$ is a bounded linear operator. The solution set of the SVIP is denoted by $\Omega:=\left\{x^{*} \in \operatorname{Sol}\left(C, F_{1}\right): A x^{*} \in \operatorname{Sol}\left(Q, F_{2}\right)\right\}$. In particular, if $F_{i}$ is $\alpha_{i}$-inverse strongly monotone on $\mathscr{H}_{i}$ for $i=1,2$, Censor, Gibali, Reich [5] devised the following iterative algorithm for solving the SVIP:

$$
\left\{\begin{array}{l}
x^{0} \in \mathscr{H}_{1} \text { chosen arbitrarily, } \\
x^{n+1}=P_{C}^{F_{1}, \lambda}\left(x^{n}-\ell A^{*}\left(I-P_{Q}^{F_{2}, \lambda}\right) A x^{n}\right), \quad \forall n \geq 0,
\end{array}\right.
$$

where $\ell \in\left(0, \frac{1}{\|A\|^{2}}\right), 0 \leq \lambda \leq 2 \min \left\{\alpha_{1}, \alpha_{2}\right\}, P_{C}^{F_{1}, \lambda}=P_{C}\left(I-\lambda F_{1}\right)$ and $P_{C}^{F_{2}, \lambda}=P_{C}\left(I-\lambda F_{2}\right)$. It was proven in [5] that the sequence $\left\{x^{n}\right\}$ is weakly convergent provided $\Omega \neq \emptyset$.

In particular, if $F_{1}=0$ and $F_{2}=0$, then the SVIP is reduced to the split feasibility problem (SFP) of finding $x^{*} \in C$ such that $A x^{*} \in Q$, which was first introduced by Censor and Elfving [6] in finite-dimensional Hilbert spaces for modeling inverse problems. It is well known that the SFP can be used to model the intensity-modulated radiation therapy $[3,7,8]$, and other real world problems. For recent results on approximation solutions of the SFP, we refer to $[9,18,21]$.

Recently, Anh [2] studied the following bilevel split variational inequality problem (BSVIP):

$$
\text { Find } x^{*} \in \Omega:\left\langle\Phi x^{*}, x-x^{*}\right\rangle \geq 0, \quad \forall x \in \Omega,
$$

where $\Omega$ is the same as above, and $\Phi: C \rightarrow \mathscr{H}_{1}$ is $\eta$-strongly monotone, $\kappa$-Lipschitzian on $C$. He introduced a linesearch extragradient method for solving the above BSVIP and gave the strong convergence analysis in the framework of Hilbert spaces.

Inspired by the results presented in [2], we introduce two modified inertial linesearch extragradient algorithms for finding a solution of the BSVIP with a fixed-point constraint. Our proposed algorithms are based on the inertial method, the linesearch extragradient method, the hybrid steepest-descent method, and the viscosity approximation method. Under some suitable 
conditions, it is proven that the sequences constructed by our proposed algorithms converge strongly to a solution of the BSVIP. The remainder of this paper is arranged below. In Section 2, some definitions and lemmas are provided for the later use. In Section 3, the convergence analysis of the proposed algorithms is investigated, and the proposed algorithms are applied to solve strongly monotone variational inequalities with constraints.

\section{PRELIMINARIES}

Let $\mathscr{H}$ be a real Hilbert space and let $C$ be a closed convex nonempty subset. Let $S: C \rightarrow \mathscr{H}$ be a nonexpansive mapping. Recall that $S$ is said to be $\delta$-contractive if $\|S x-S y\| \leq \delta\|x-y\|$, $\forall x, y \in C$, where $\delta$ is a constant in $(0,1)$. $S$ is said to be nonexpansive if $\|S x-S y\| \leq\|x-y\|$, $\forall x, y \in C$. $S$ is said to be quasi-nonexpansive if $\operatorname{Fix}(S) \neq \emptyset$, and $\|S x-y\| \leq\|x-y\|, \forall x \in C$, $y \in \operatorname{Fix}(S)$. The class of quasi-nonexpansive is quite different from the class of nonexpansive. Indeed, quasi-nonexpansive mappings may not be continuous.

Next, we give an example of a quasi-nonexpansive mapping, which is not nonexpansive.

Example 2.1. Let $\mathscr{H}=\mathbb{R}$ with the inner product $\langle a, b\rangle=a b$ and induced norm $\|\cdot\|=|\cdot|$. Let $S: \mathscr{H} \rightarrow \mathscr{H}$ be defined by $S x:=\frac{2 x}{3} \sin x, \forall x \in \mathscr{H}$. We first claim that $\operatorname{Fix}(S)=\{0\}$. Indeed, if $x \neq 0$ and $S x=x$, then $\sin x=\frac{3}{2}$, which reaches a contradiction. Hence we get $\operatorname{Fix}(S)=\{0\}$. Also, we claim that $S$ is quasi-nonexpansive. Indeed, we note that $\|S x-0\|=\left\|\frac{2 x}{3} \sin x\right\| \leq$ $\left\|\frac{2 x}{3}\right\| \leq\|x\|=\|x-0\|, \forall x \in \mathscr{H}$. However, we claim that $S$ is not nonexpansive. In fact, setting $x=2 \pi$ and $y=\frac{3 \pi}{2}$, we get $\|S x-S y\|=\pi>\left\|2 \pi-\frac{3 \pi}{2}\right\|=\frac{\pi}{2}$.

The demiclosed principle is a useful tool in study of fixed points of nonexpansive mappings. Let $S: C \rightarrow \mathscr{H}$ be a mapping with $\operatorname{Fix}(S) \neq \emptyset$. Recall that $I-S$ is said to be demiclosed at zero if, for any $\left\{x^{n}\right\} \subset C$ with $x^{n} \rightarrow x,(I-S) x^{n} \rightarrow 0 \Rightarrow x \in \operatorname{Fix}(S)$. It is known that the class of nonexpansive mappings process the demiclosed principle. However, the class of quasinonexpansive mappings may do not process the demiclosed principle; see, e.g., [23, 27].

The following lemmas play an important role in the proof of our main convergence theorems.

Lemma 2.1. [11] Let $F: C \rightarrow \mathscr{H}$ be a pseudomonotone and continuous mapping. For any $x \in C$, we have $\langle F x, y-x\rangle \geq 0, \Leftrightarrow\langle F y, y-x\rangle \geq 0, \forall y \in C$.

Lemma 2.2. [26] Let $\left\{a_{n}\right\}$ be a sequence in $[0,+\infty)$ satisfying the condition: $a_{n+1} \leq(1-$ $\left.s_{n}\right) a_{n}+s_{n} b_{n}, \forall n \geq 0$, where $\left\{s_{n}\right\}$ and $\left\{b_{n}\right\}$ are real sequences such that $(a)\left\{s_{n}\right\} \subset[0,1]$ and $\sum_{n=0}^{\infty} s_{n}=\infty$, and $(b) \limsup _{n \rightarrow \infty} b_{n} \leq 0$ or $\sum_{n=0}^{\infty}\left|s_{n} b_{n}\right|<\infty$. Then $\lim _{n \rightarrow \infty} a_{n}=0$.

Lemma 2.3. [13] Let $S$ be a nonexpansive self-mapping on $C$ with $\operatorname{Fix}(S) \neq \emptyset$. Then $I-S$ is demiclosed at zero.

Lemma 2.4. [26] Let $\ell \in(0,1], S: C \rightarrow C$ be a nonexpansive mapping, and the mapping $S^{\ell}$ : $C \rightarrow \mathscr{H}$ be defined by $S^{\ell} x:=S x-\ell \mu F(S x), \forall x \in C$, where $F: C \rightarrow \mathscr{H}$ is $\kappa$-Lipschitzian and $\eta$-strongly monotone. If $0<\mu<\frac{2 \eta}{\kappa^{2}}$, then $S^{\ell}$ is a contraction, that is, $\left\|S^{\ell} x-S^{\ell} y\right\| \leq$ $(1-\ell \tau)\|x-y\|, \forall x, y \in C$, where $\tau=1-\sqrt{1-\mu\left(2 \eta-\mu \kappa^{2}\right)} \in(0,1]$.

Lemma 2.5. [16] Let $\left\{\Gamma_{n}\right\}$ be a sequence of real numbers that does not decrease at infinity in the sense that there exists a subsequence $\left\{\Gamma_{n_{k}}\right\}$ of $\left\{\Gamma_{n}\right\}$ which satisfies $\Gamma_{n_{k}}<\Gamma_{n_{k}+1}$ for each integer $k \geq 1$. Define the sequence $\{\tau(n)\}_{n \geq n_{0}}$ of integers as follows: $\tau(n)=\max \left\{k \leq n: \Gamma_{k}<\right.$ $\left.\Gamma_{k+1}\right\}$, where integer $n_{0} \geq 1$ such that $\left\{k \leq n_{0}: \Gamma_{k}<\Gamma_{k+1}\right\} \neq \emptyset$. Then, the following hold: 
(i) $\tau\left(n_{0}\right) \leq \tau\left(n_{0}+1\right) \leq \cdots$ and $\tau(n) \rightarrow \infty$;

(ii) $\Gamma_{\tau(n)} \leq \Gamma_{\tau(n)+1}$ and $\Gamma_{n} \leq \Gamma_{\tau(n)+1}, \forall n \geq n_{0}$.

\section{MAIN RESULTS}

In this section, we give two strong convergence algorithms for solving the BSVIP with the fixed-point constraint of a quasi-nonexpansive mapping by using the modified inertial linesearch extragradient methods. In what follows, we always assume the conditions:

(A1) $F: C \rightarrow \mathscr{H}_{1}$ is pseudomonotone on $C$,

(A2) $\lim _{k \rightarrow \infty} F x^{k}=F \bar{x}$ for every sequence $\left\{x^{k}\right\} \subset C$ converging weakly to $\bar{x}$,

(A3) $G: Q \rightarrow \mathscr{H}_{2}$ is pseudomonotone on $Q$,

(A4) $\lim _{k \rightarrow \infty} G u^{k}=G \bar{u}$ for every sequence $\left\{u^{k}\right\} \subset Q$ converging weakly to $\bar{u}$,

(A5) $f: C \rightarrow \mathscr{H}_{1}$ is a $\delta$-contractive mapping on $C$ and $\Phi: C \rightarrow \mathscr{H}_{1}$ is $\kappa$-Lipschitzian, $\eta$ strongly monotone mapping on $C$, where $\delta<\tau:=1-\sqrt{1-\mu\left(2 \eta-\mu \kappa^{2}\right)}$ for $\mu \in$ $\left(0, \frac{2 \eta}{\kappa^{2}}\right)$,

(A6) $T$ is a quasi-nonexpansive self-mapping on $C$ and $I-T$ is demiclosed at zero such that $\operatorname{Fix}(T) \cap \Omega \neq \emptyset$,

(A7) $\left\{\varepsilon_{k}\right\} \subset(0,1]$ and $\left\{\beta_{k}\right\},\left\{v_{k}\right\} \subset(0,1)$ such that

(i) $\beta_{k}+v_{k} \leq 1, \sum_{k=1}^{\infty} \beta_{k}=\infty, \lim _{k \rightarrow \infty} \beta_{k}=0$,

(ii) $\varepsilon_{k}=o\left(\beta_{k}\right)$, i.e., $\lim _{k \rightarrow \infty} \frac{\varepsilon_{k}}{\beta_{k}}=0$,

(iii) $0<\liminf _{k \rightarrow \infty} v_{k} \leq \limsup _{k \rightarrow \infty} v_{k}<1$.

Algorithm 3.1. Initial Step: Give $x^{0}, x^{1} \in C$ arbitrarily. Let $\alpha>0, \ell \in(0,1), \gamma \in(0,1)$, $\left\{\boldsymbol{\delta}_{k}\right\} \subset[\underline{\boldsymbol{\delta}}, \overline{\boldsymbol{\delta}}] \subset\left(0, \frac{1}{1+\|A\|^{2}}\right),\left\{\lambda_{k}\right\} \subset(0,1)$, and $\lim _{k \rightarrow \infty} \lambda_{k}=\lambda \in(0,1)$.

Iteration Steps: Compute $x^{k+1}$ below:

Step 1. given the iterates $x^{k-1}$ and $x^{k}(k \geq 1)$, choose $\alpha_{k}$ such that $0 \leq \alpha_{k} \leq \bar{\alpha}_{k}$, where

$$
\bar{\alpha}_{k}= \begin{cases}\min \left\{\alpha, \frac{\varepsilon_{k}}{\left\|x^{k}-x^{k-1}\right\|}\right\}, & \text { if } x^{k}-x^{k-1} \neq 0, \\ \alpha, & \text { otherwise. }\end{cases}
$$

Step 2. Set $p^{k}=x^{k}+\alpha_{k}\left(x^{k}-x^{k-1}\right)$, and calculate $u^{k}=P_{Q}\left(A p^{k}\right)$ and $v^{k}=P_{Q}\left(u^{k}-G u^{k}\right)$. If $v^{k}=u^{k}$, then set $t^{k}=u^{k}$ and go to Step 5. Otherwise, go to Step 3 .

Step 3. Find $i_{k}$ as the smallest nonnegative integer $i$ such that $w^{k, i}=\left(1-\gamma^{i}\right) u^{k}+\gamma^{i} v^{k}$ and $\left\langle G w^{k, i}, u^{k}-v^{k}\right\rangle \geq \frac{1}{2}\left\|u^{k}-v^{k}\right\|^{2}$. Set $\gamma_{k}=\gamma^{i_{k}}$ and $w^{k}=w^{k, i_{k}}$.

Step 4. Calculate $t^{k}=P_{Q}\left(u^{k}-\sigma_{k} G w^{k}\right)$, where $\sigma_{k}=\frac{\left\langle G w^{k}, u^{k}-w^{k}\right\rangle}{\left\|G w^{k}\right\|^{2}}$.

Step 5. Calculate $\bar{u}^{k}=P_{C}\left(p^{k}+\delta_{k} A^{*}\left(t^{k}-A p^{k}\right)\right)$ and $\bar{v}^{k}=P_{C}\left(\bar{u}^{k}-F \bar{u}^{k}\right)$. If $\bar{v}^{k}=\bar{u}^{k}$, then set $y^{k}=\bar{u}^{k}$ and go to Step 8. Otherwise, go to Step 6 .

Step 6. Find $j_{k}$ as the smallest nonnegative integer $j$ such that $\bar{w}^{k, j}=\left(1-\ell^{j}\right) \bar{u}^{k}+\ell^{j} \bar{v}^{k}$ and $\left\langle F \bar{w}^{k, j}, \bar{u}^{k}-\bar{v}^{k}\right\rangle \geq \frac{1}{2}\left\|\bar{u}^{k}-\bar{v}^{k}\right\|^{2}$. Set $\ell_{k}=\ell^{j_{k}}$ and $\bar{w}^{k}=\bar{w}^{k, j_{k}}$.

Step 7. Calculate $y^{k}=P_{C}\left(\bar{u}^{k}-\bar{\sigma}_{k} F \bar{w}^{k}\right)$, where $\bar{\sigma}_{k}=\frac{\left\langle F \bar{w}^{k} \bar{u}^{k}-\bar{w}^{k}\right\rangle}{\left\|F \bar{w}^{k}\right\|^{2}}$.

Step 8. Calculate $z^{k}=\left(1-\lambda_{k}\right) \bar{u}^{k}+\lambda_{k} y^{k}$ and

$$
x^{k+1}=P_{C}\left[\beta_{k} f\left(z^{k}\right)+v_{k} p^{k}+\left(\left(1-v_{k}\right) I-\beta_{k} \mu \Phi\right) T z^{k}\right] .
$$

Set $k:=k+1$ and go to Step 1 . 
Remark 3.1. (i) In finite dimensional spaces, conditions (A2) and (A4) are reduced to the conditions for the continuity of $F, G$.

(ii) If $F$ and $G$ satisfy properties (A1), (A2) and (A3), (A4) respectively, then the solution sets $\operatorname{Sol}(C, F)$ and $\operatorname{Sol}(Q, G)$ of the $\operatorname{VIP}(C, F)$ and $\operatorname{VIP}(Q, G)$ are closed and convex (see e.g., [1, Lemma 6]). Therefore, the solution set $\Omega=\left\{x^{*} \in \operatorname{Sol}(C, F): A x^{*} \in \operatorname{Sol}(Q, G)\right\}$ of the SVIP is also closed and convex.

(iii) If $\left\{x^{k}\right\} \subset C$ is bounded, then $\left\{F x^{k}\right\}$ is bounded. In fact, assume that $\left\{F x^{k}\right\}$ is unbounded, that is, $\exists\left\{x^{k_{i}}\right\} \subset\left\{x^{k}\right\}$ such that $\left\|F x^{k_{i}}\right\| \rightarrow \infty(i \rightarrow \infty)$. But the boundedness of $\left\{x^{k_{i}}\right\}$ guarantees that $\exists\left\{x^{k_{i_{l}}}\right\} \subset\left\{x^{k_{i}}\right\}$ such that $x^{k_{i_{l}}} \rightarrow \hat{x}$. Hence $F x^{k_{i_{l}}} \rightarrow F \hat{x}(l \rightarrow \infty)$. Thus, $\left\|F x^{k_{i_{l}}}\right\| \rightarrow\|F \hat{x}\|(l \rightarrow$ $\infty)$. From $\left\|F x^{k_{i}}\right\| \rightarrow \infty(i \rightarrow \infty)$, we get $\left\|F x^{k_{i}}\right\| \rightarrow \infty(l \rightarrow \infty)$, a contradiction. Consequently, $\left\{F x^{k}\right\}$ is bounded.

(iv) $\lim _{k \rightarrow \infty} \frac{\alpha_{k}\left\|x^{k}-x^{k-1}\right\|}{\beta_{k}}=0$. In fact, we get from (3.1) that $\varepsilon_{k} \geq \alpha_{k}\left\|x^{k}-x^{k-1}\right\|, \forall k \geq 1$. Since $\lim _{k \rightarrow \infty} \frac{\varepsilon_{k}}{\beta_{k}}=0$, we have $0=\lim _{k \rightarrow \infty} \frac{\varepsilon_{k}}{\beta_{k}} \geq \lim \sup _{k \rightarrow \infty} \frac{\alpha_{k}\left\|x^{k}-x^{k-1}\right\|}{\beta_{k}}$.

We are now in a position to state and prove the first main result in this paper.

Theorem 3.1. Let $\left\{x^{k}\right\}$ be the sequence defined by Algorithm 3.1. Then $x^{k} \rightarrow x^{*} \in \operatorname{Fix}(T) \cap \Omega$, which is a unique solution to the VIP: $\left\langle(\mu \Phi-f) x^{*}, p-x^{*}\right\rangle \geq 0, \forall p \in \operatorname{Fix}(T) \cap \Omega$.

Proof. We first claim that $P_{\operatorname{Fix}(T) \cap \Omega}(f+I-\mu \Phi)$ is a contraction. Indeed, using Lemma 2.4, we have

$$
\begin{aligned}
& \left\|P_{\operatorname{Fix}(T) \cap \Omega}(f+I-\mu \Phi) x-P_{\operatorname{Fix}(T) \cap \Omega}(f+I-\mu \Phi) y\right\| \\
\leq & \|f(x)-f(y)\|+\|(I-\mu \Phi) x-(I-\mu \Phi) y\| \\
\leq & {[1-(\tau-\delta)]\|x-y\| \quad \forall x, y \in \mathscr{H}_{1}, }
\end{aligned}
$$

which implies that $P_{\mathrm{Fix}(T) \cap \Omega}(f+I-\mu \Phi)$ is a contraction. Banach's Contraction Mapping Principle guarantees that $P_{\mathrm{Fix}(T) \cap \Omega}(f+I-\mu \Phi)$ has a unique fixed point, say $x^{*} \in \mathscr{H}_{1}$, that is, $x^{*}=P_{\operatorname{Fix}(T) \cap \Omega}(f+I-\mu \Phi) x^{*}$. Thus, there is an unique solution $x^{*} \in \operatorname{Fix}(T) \cap \Omega$ to the VIP

$$
\left\langle(\mu \Phi-f) x^{*}, p-x^{*}\right\rangle \geq 0 \quad \forall p \in \operatorname{Fix}(T) \cap \Omega .
$$

The following proof is split into eight steps.

Step 1. Show that the linesearches corresponding to $u^{k}, v^{k}$ and $\bar{u}^{k}, \bar{v}^{k}$ are well defined, respectively.

Indeed, in the case of $v^{k} \neq u^{k}$, we assume that the following inequality holds for each integer $i \geq 1$

$$
\left\langle G w^{k, i}, u^{k}-v^{k}\right\rangle<\frac{1}{2}\left\|u^{k}-v^{k}\right\|^{2},
$$

where $w^{k, i}=\left(1-\gamma^{i}\right) u^{k}+\gamma^{i} v^{k}$. Since $w^{k, i} \rightarrow u^{k}$ as $i \rightarrow \infty$, it follows that

$$
\left\langle G u^{k}, u^{k}-v^{k}\right\rangle \leq \frac{1}{2}\left\|u^{k}-v^{k}\right\|^{2} .
$$

In view of $v^{k}=P_{Q}\left(u^{k}-G u^{k}\right)$, we get

$$
\left\langle u^{k}-G u^{k}-v^{k}, u-v^{k}\right\rangle \leq 0 \quad \forall u \in Q .
$$

Putting $u=u^{k} \in Q$, we have

$$
\left\langle G u^{k}, u^{k}-v^{k}\right\rangle \geq\left\|u^{k}-v^{k}\right\|^{2}
$$


This together with (3.3) leads to

$$
\left\|u^{k}-v^{k}\right\|^{2} \leq \frac{1}{2}\left\|u^{k}-v^{k}\right\|^{2}
$$

which contradicts the fact that $u^{k} \neq v^{k}$. Consequently, the linesearch corresponding to $u^{k}$ and $v^{k}$ is well defined. Similarly, we know that the linesearch corresponding to $\bar{u}^{k}$ and $\bar{v}^{k}$ is also well defined.

Step 2. Show that the following statements hold.

(a) If $v^{k} \neq u^{k}$ for some $k \geq 0$, then $G w^{k} \neq 0, \sigma_{k}>0$ and

$$
\left\|t^{k}-A x^{*}\right\|^{2} \leq\left\|u^{k}-A x^{*}\right\|^{2}-\left(\sigma_{k}\left\|G w^{k}\right\|\right)^{2} .
$$

(b) If $\bar{v}^{k} \neq \bar{u}^{k}$ for some $k \geq 0$, then $F \bar{w}^{k} \neq 0, \bar{\sigma}_{k}>0$ and

$$
\left\|y^{k}-x^{*}\right\|^{2} \leq\left\|\bar{u}^{k}-x^{*}\right\|^{2}-\left(\bar{\sigma}_{k}\left\|F \bar{w}^{k}\right\|\right)^{2} .
$$

Indeed, in the case of $v^{k} \neq u^{k}$, we get

$$
\left\langle G w^{k}, u^{k}-w^{k}\right\rangle=\gamma^{i}\left\langle G w^{k}, u^{k}-v^{k}\right\rangle \geq \frac{\gamma^{i}\left\|u^{k}-v^{k}\right\|^{2}}{2}>0,
$$

which hence yields

$$
G w^{k} \neq 0, \quad \sigma_{k}=\frac{\left\langle G w^{k}, u^{k}-w^{k}\right\rangle}{\left\|G w^{k}\right\|^{2}}>0 .
$$

Since $A x^{*} \in \operatorname{Sol}(Q, G)$ and $w^{k} \in Q$, we get $\left\langle G A x^{*}, w^{k}-A x^{*}\right\rangle \geq 0$. Utilizing the pseudomonotonicity of $G$, we have

$$
\left\langle G w^{k}, w^{k}-A x^{*}\right\rangle \geq 0
$$

This further yields

$$
\begin{aligned}
\left\|t^{k}-A x^{*}\right\|^{2} & \leq\left\|u^{k}-\sigma_{k} G w^{k}-A x^{*}\right\|^{2} \\
& =\left\|u^{k}-A x^{*}\right\|^{2}+\sigma_{k}^{2}\left\|G w^{k}\right\|^{2}-2 \sigma_{k}\left\langle G w^{k}, u^{k}-A x^{*}\right\rangle \\
& \leq\left\|u^{k}-A x^{*}\right\|^{2}+\sigma_{k}^{2}\left\|G w^{k}\right\|^{2}-2 \sigma_{k}\left\langle G w^{k}, u^{k}-w^{k}\right\rangle \\
& =\left\|u^{k}-A x^{*}\right\|^{2}-\left(\sigma_{k}\left\|G w^{k}\right\|\right)^{2} .
\end{aligned}
$$

Similarly, we can obtain (b).

Step 3. Show that

$$
\left\|\bar{u}^{k}-x^{*}\right\|^{2} \leq\left\|p^{k}-x^{*}\right\|^{2}-\delta_{k}\left(1-\delta_{k}\|A\|^{2}\right)\left\|t^{k}-A p^{k}\right\|^{2}-\delta_{k}\left\|u^{k}-A p^{k}\right\|^{2} \quad \forall k \geq 1 .
$$

Indeed, in terms of Step 2 (a) and the fact that $t^{k}=u^{k}$ if $v^{k}=u^{k}$, we get

$$
\left\|t^{k}-A x^{*}\right\| \leq\left\|u^{k}-A x^{*}\right\| \text {. }
$$

Note that

$$
\begin{aligned}
\left\|u^{k}-A x^{*}\right\|^{2}=\left\|P_{Q} A p^{k}-A x^{*}\right\|^{2} & \leq\left\|A p^{k}-A x^{*}\right\|^{2}-\left\|P_{Q} A p^{k}-A p^{k}\right\|^{2} \\
& =\left\|A p^{k}-A x^{*}\right\|^{2}-\left\|u^{k}-A p^{k}\right\|^{2}
\end{aligned}
$$

Combining (3.4) and (3.5) yields

$$
\left\|t^{k}-A x^{*}\right\|^{2}-\left\|A p^{k}-A x^{*}\right\|^{2} \leq-\left\|u^{k}-A p^{k}\right\|^{2}
$$


from which it follows that

$$
\begin{aligned}
\left\langle A\left(p^{k}-x^{*}\right), t^{k}-A p^{k}\right\rangle & =\left\langle t^{k}-A x^{*}, t^{k}-A p^{k}\right\rangle-\left\|t^{k}-A p^{k}\right\|^{2} \\
& =\frac{1}{2}\left[\left(\left\|t^{k}-A x^{*}\right\|^{2}-\left\|A p^{k}-A x^{*}\right\|^{2}\right)-\left\|t^{k}-A p^{k}\right\|^{2}\right] \\
& \leq-\frac{1}{2}\left(\left\|u^{k}-A p^{k}\right\|^{2}+\left\|t^{k}-A p^{k}\right\|^{2}\right) .
\end{aligned}
$$

From $\delta_{k}>0$ and the last inequality, we get

$$
2 \delta_{k}\left\langle A\left(p^{k}-x^{*}\right), t^{k}-A p^{k}\right\rangle \leq-\delta_{k}\left(\left\|u^{k}-A p^{k}\right\|^{2}+\left\|t^{k}-A p^{k}\right\|^{2}\right),
$$

which together with the nonexpansivity of $P_{C}$ yields

$$
\begin{aligned}
\left\|\bar{u}^{k}-x^{*}\right\|^{2} & \leq\left\|p^{k}-x^{*}\right\|^{2}+\delta_{k}^{2}\left\|A^{*}\left(t^{k}-A p^{k}\right)\right\|^{2}+2 \delta_{k}\left\langle p^{k}-x^{*}, A^{*}\left(t^{k}-A p^{k}\right)\right\rangle \\
& \left.\leq\left\|p^{k}-x^{*}\right\|^{2}+\delta_{k}^{2}\left\|A^{*}\right\|^{2}\left\|t^{k}-A p^{k}\right\|^{2}+2 \delta_{k}\left\langle A\left(p^{k}-x^{*}\right), t^{k}-A p^{k}\right)\right\rangle \\
& \leq\left\|p^{k}-x^{*}\right\|^{2}+\delta_{k}^{2}\|A\|^{2}\left\|t^{k}-A p^{k}\right\|^{2}-\delta_{k}\left(\left\|u^{k}-A p^{k}\right\|^{2}+\left\|t^{k}-A p^{k}\right\|^{2}\right) \\
& =\left\|p^{k}-x^{*}\right\|^{2}-\delta_{k}\left(1-\delta_{k}\|A\|^{2}\right)\left\|t^{k}-A p^{k}\right\|^{2}-\delta_{k}\left\|u^{k}-A p^{k}\right\|^{2} .
\end{aligned}
$$

Step 4. Show that

$$
\left\|z^{k}-x^{*}\right\|^{2} \leq\left\|\bar{u}^{k}-x^{*}\right\|^{2}-\lambda_{k}\left(1-\lambda_{k}\right)\left\|y^{k}-\bar{u}^{k}\right\|^{2} \quad \forall k \geq 1 .
$$

Indeed, from $y^{k}=\bar{u}^{k}$ if $\bar{v}^{k}=\bar{u}^{k}$ and Step 2 (b), we get

$$
\left\|y^{k}-x^{*}\right\| \leq\left\|\bar{u}^{k}-x^{*}\right\|
$$

which hence implies that

$$
\begin{aligned}
\left\|z^{k}-x^{*}\right\|^{2} & =\left(1-\lambda_{k}\right)\left\|\bar{u}^{k}-x^{*}\right\|^{2}+\lambda_{k}\left\|y^{k}-x^{*}\right\|^{2}-\lambda_{k}\left(1-\lambda_{k}\right)\left\|y^{k}-\bar{u}^{k}\right\|^{2} \\
& \leq\left(1-\lambda_{k}\right)\left\|\bar{u}^{k}-x^{*}\right\|^{2}+\lambda_{k}\left\|\bar{u}^{k}-x^{*}\right\|^{2}-\lambda_{k}\left(1-\lambda_{k}\right)\left\|y^{k}-\bar{u}^{k}\right\|^{2} \\
& =\left\|\bar{u}^{k}-x^{*}\right\|^{2}-\lambda_{k}\left(1-\lambda_{k}\right)\left\|y^{k}-\bar{u}^{k}\right\|^{2} .
\end{aligned}
$$

Step 5. Show that $\left\{x^{k}\right\}$ is bounded.

Indeed, since $\left\{\delta_{k}\right\} \subset[\underline{\delta}, \bar{\delta}] \subset\left(0, \frac{1}{1+\|A\|^{2}}\right)$ and $\left\{\lambda_{k}\right\} \subset(0,1)$, we get

$$
\left\|z^{k}-x^{*}\right\| \leq\left\|\bar{u}^{k}-x^{*}\right\| \leq\left\|p^{k}-x^{*}\right\| \quad \forall k \geq 1 .
$$

From the definition of $p^{k}$, we have

$$
\begin{aligned}
\left\|p^{k}-x^{*}\right\| & =\left\|x^{k}+\alpha_{k}\left(x^{k}-x^{k-1}\right)-x^{*}\right\| \\
& \leq\left\|x^{k}-x^{*}\right\|+\beta_{k} \cdot \frac{\alpha_{k}}{\beta_{k}}\left\|x^{k}-x^{k-1}\right\| .
\end{aligned}
$$

According to Remark 3.1 (iv), we have $\frac{\alpha_{k}}{\beta_{k}}\left\|x^{k}-x^{k-1}\right\| \rightarrow 0(k \rightarrow \infty)$. So it follows that there exists a constant $M_{0}>0$ such that

$$
\frac{\alpha_{k}}{\beta_{k}}\left\|x^{k}-x^{k-1}\right\| \leq M_{0} \quad \forall k \geq 1
$$

Combining this with (3.8) and (3.9), we get

$$
\left\|z^{k}-x^{*}\right\| \leq\left\|\bar{u}^{k}-x^{*}\right\| \leq\left\|p^{k}-x^{*}\right\| \leq\left\|x^{k}-x^{*}\right\|+\beta_{k} M_{0} \quad \forall k \geq 1 .
$$


Thus, from $\beta_{k}+v_{k} \leq 1$, Lemma 2.4 and (3.10), we conclude that, for all $k \geq 1$,

$$
\begin{aligned}
\left\|x^{k+1}-x^{*}\right\| \leq & \beta_{k}\left(\left\|f\left(z^{k}\right)-f\left(x^{*}\right)\right\|+\left\|f\left(x^{*}\right)-x^{*}\right\|\right)+v_{k}\left\|p^{k}-x^{*}\right\| \\
& +\left(1-\beta_{k}-v_{k}\right)\left\|\left(\frac{1-v_{k}}{1-\beta_{k}-v_{k}} I-\frac{\beta_{k}}{1-\beta_{k}-v_{k}} \mu \Phi\right) T z^{k}-x^{*}\right\| \\
\leq & \beta_{k}\left(\delta\left\|z^{k}-x^{*}\right\|+\left\|f\left(x^{*}\right)-x^{*}\right\|\right)+v_{k}\left\|p^{k}-x^{*}\right\| \\
& +\left(1-v_{k}\right)\left\|\left(I-\frac{\beta_{k}}{1-v_{k}} \mu \Phi\right) T z^{k}-\left(I-\frac{\beta_{k}}{1-v_{k}} \mu \Phi\right) x^{*}+\frac{\beta_{k}}{1-v_{k}}(I-\mu \Phi) x^{*}\right\| \\
\leq & \beta_{k}\left(\delta\left\|z^{k}-x^{*}\right\|+\left\|f\left(x^{*}\right)-x^{*}\right\|\right)+v_{k}\left\|p^{k}-x^{*}\right\| \\
& +\left(1-v_{k}\right)\left[\left(1-\frac{\beta_{k}}{1-v_{k}} \tau\right)\left\|z^{k}-x^{*}\right\|+\frac{\beta_{k}}{1-v_{k}}\left\|(I-\mu \Phi) x^{*}\right\|\right] \\
\leq & {\left[1-\beta_{k}(\tau-\delta)\right]\left\|p^{k}-x^{*}\right\|+\beta_{k}\left(\left\|f\left(x^{*}\right)-x^{*}\right\|+\left\|(I-\mu \Phi) x^{*}\right\|\right) } \\
\leq & {\left[1-\beta_{k}(\tau-\delta)\right]\left(\left\|x^{k}-x^{*}\right\|+\beta_{k} M_{0}\right)+\beta_{k}\left(\left\|f\left(x^{*}\right)-x^{*}\right\|+\left\|(I-\mu \Phi) x^{*}\right\|\right) } \\
\leq & {\left[1-\beta_{k}(\tau-\delta)\right]\left\|x^{k}-x^{*}\right\|+\beta_{k}\left(M_{0}+\left\|f\left(x^{*}\right)-x^{*}\right\|+\left\|(I-\mu \Phi) x^{*}\right\|\right) } \\
\leq & \max \left\{\left\|x^{k}-x^{*}\right\|, \frac{M_{0}+\left\|f\left(x^{*}\right)-x^{*}\right\|+\left\|(I-\mu \Phi) x^{*}\right\|}{\tau-\delta}\right\} .
\end{aligned}
$$

By induction, we obtain $\left\|x^{k}-x^{*}\right\| \leq \max \left\{\left\|x^{1}-x^{*}\right\|, \frac{M_{0}+\left\|f\left(x^{*}\right)-x^{*}\right\|+\left\|(I-\mu \Phi) x^{*}\right\|}{\tau-\delta}\right\}$. Thus, $\left\{x^{k}\right\}$ is bounded, so are $\left\{p^{k}\right\},\left\{\bar{u}^{k}\right\},\left\{z^{k}\right\},\left\{f\left(z^{k}\right)\right\},\left\{T z^{k}\right\}$, and $\left\{\Phi T z^{k}\right\}$.

Step 6. Show that

$$
\begin{aligned}
\left\|x^{k+1}-x^{*}\right\|^{2} \leq & {\left[1-\beta_{k}(\tau-\delta)\right]\left\|x^{k}-x^{*}\right\|^{2}+\beta_{k}(\tau-\delta)\left[\frac{2\left\langle(f-\mu \Phi) x^{*}, x^{k+1}-x^{*}\right\rangle}{\tau-\delta}\right.} \\
& \left.+\frac{3 M}{\tau-\delta} \frac{\alpha_{k}}{\beta_{k}}\left\|x^{k}-x^{k-1}\right\|\right]
\end{aligned}
$$

for some $M>0$. Putting $\omega^{k}:=\beta_{k} f\left(z^{k}\right)+v_{k} p^{k}+\left(\left(1-v_{k}\right) I-\beta_{k} \mu \Phi\right) T z^{k}$, we have $x^{k+1}=P_{C} \omega^{k}$. Note that

$$
\begin{aligned}
\left\|x^{k+1}-x^{*}\right\|^{2} \leq & \left\langle\beta_{k}\left(f\left(z^{k}\right)-f\left(x^{*}\right)\right)+v_{k}\left(p^{k}-x^{*}\right)+\left(1-v_{k}\right)\left[\left(I-\frac{\beta_{k}}{1-v_{k}} \mu \Phi\right) T z^{k}\right.\right. \\
& \left.\left.-\left(I-\frac{\beta_{k}}{1-v_{k}} \mu \Phi\right) x^{*}\right], x^{k+1}-x^{*}\right\rangle+\beta_{k}\left\langle(f-\mu \Phi) x^{*}, x^{k+1}-x^{*}\right\rangle \\
\leq & \| \beta_{k}\left(f\left(z^{k}\right)-f\left(x^{*}\right)\right)+v_{k}\left(p^{k}-x^{*}\right)+\left(1-v_{k}\right)\left[\left(I-\frac{\beta_{k}}{1-v_{k}} \mu \Phi\right) T z^{k}\right. \\
& \left.-\left(I-\frac{\beta_{k}}{1-v_{k}} \mu \Phi\right) x^{*}\right]\|\| x^{k+1}-x^{*} \|+\beta_{k}\left\langle(f-\mu \Phi) x^{*}, x^{k+1}-x^{*}\right\rangle \\
\leq & \frac{1}{2}\left\{\| \beta_{k}\left(f\left(z^{k}\right)-f\left(x^{*}\right)\right)+v_{k}\left(p^{k}-x^{*}\right)+\left(1-v_{k}\right)\left[\left(I-\frac{\beta_{k}}{1-v_{k}} \mu \Phi\right) T z^{k}\right.\right. \\
& \left.\left.-\left(I-\frac{\beta_{k}}{1-v_{k}} \mu \Phi\right) x^{*}\right]\left\|^{2}+\right\| x^{k+1}-x^{*} \|^{2}\right\}+\beta_{k}\left\langle(f-\mu \Phi) x^{*}, x^{k+1}-x^{*}\right\rangle .
\end{aligned}
$$


Utilizing Lemma 2.4 and (3.10), we obtain that

$$
\begin{aligned}
\left\|x^{k+1}-x^{*}\right\|^{2} \leq & \| \beta_{k}\left(f\left(z^{k}\right)-f\left(x^{*}\right)\right)+v_{k}\left(p^{k}-x^{*}\right)+\left(1-v_{k}\right)\left[\left(I-\frac{\beta_{k}}{1-v_{k}} \mu \Phi\right) T z^{k}\right. \\
& \left.-\left(I-\frac{\beta_{k}}{1-v_{k}} \mu \Phi\right) x^{*}\right] \|^{2}+2 \beta_{k}\left\langle(f-\mu \Phi) x^{*}, x^{k+1}-x^{*}\right\rangle \\
\leq & {\left[\beta_{k} \delta\left\|z^{k}-x^{*}\right\|+v_{k}\left\|p^{k}-x^{*}\right\|+\left(1-\beta_{k} \tau-v_{k}\right)\left\|z^{k}-x^{*}\right\|\right]^{2} } \\
& +2 \beta_{k}\left\langle(f-\mu \Phi) x^{*}, x^{k+1}-x^{*}\right\rangle \\
\leq & \beta_{k} \delta\left\|z^{k}-x^{*}\right\|^{2}+v_{k}\left\|p^{k}-x^{*}\right\|^{2}+\left(1-\beta_{k} \tau-v_{k}\right)\left\|z^{k}-x^{*}\right\|^{2} \\
& +2 \beta_{k}\left\langle(f-\mu \Phi) x^{*}, x^{k+1}-x^{*}\right\rangle \\
\leq & {\left[1-\beta_{k}(\tau-\delta)\right]\left\|p^{k}-x^{*}\right\|^{2}+2 \beta_{k}\left\langle(f-\mu \Phi) x^{*}, x^{k+1}-x^{*}\right\rangle . }
\end{aligned}
$$

From the definition of $p^{k}$, we have

$$
\begin{aligned}
\left\|p^{k}-x^{*}\right\|^{2} & \leq\left[\left\|x^{k}-x^{*}\right\|+\alpha_{k}\left\|x^{k}-x^{k-1}\right\|\right]^{2} \\
& =\left\|x^{k}-x^{*}\right\|^{2}+\alpha_{k}\left\|x^{k}-x^{k-1}\right\|\left[2\left\|x^{k}-x^{*}\right\|+\alpha_{k}\left\|x^{k}-x^{k-1}\right\|\right],
\end{aligned}
$$

which together with (3.11) implies that

$$
\begin{aligned}
& \left\|x^{k+1}-x^{*}\right\|^{2} \leq\left[1-\beta_{k}(\tau-\delta)\right]\left\|p^{k}-x^{*}\right\|^{2}+2 \beta_{k}\left\langle(f-\mu \Phi) x^{*}, x^{k+1}-x^{*}\right\rangle \\
\leq & {\left[1-\beta_{k}(\tau-\delta)\right]\left\{\left\|x^{k}-x^{*}\right\|^{2}+\alpha_{k}\left\|x^{k}-x^{k-1}\right\|\left[2\left\|x^{k}-x^{*}\right\|+\alpha_{k}\left\|x^{k}-x^{k-1}\right\|\right]\right\} } \\
& +2 \beta_{k}\left\langle(f-\mu \Phi) x^{*}, x^{k+1}-x^{*}\right\rangle \\
\leq & {\left[1-\beta_{k}(\tau-\delta)\right]\left\|x^{k}-x^{*}\right\|^{2}+\alpha_{k}\left\|x^{k}-x^{k-1}\right\|\left[2\left\|x^{k}-x^{*}\right\|+\alpha_{k}\left\|x^{k}-x^{k-1}\right\|\right] } \\
& +2 \beta_{k}\left\langle(f-\mu \Phi) x^{*}, x^{k+1}-x^{*}\right\rangle \\
\leq & {\left[1-\beta_{k}(\tau-\delta)\right]\left\|x^{k}-x^{*}\right\|^{2}+\alpha_{k}\left\|x^{k}-x^{k-1}\right\| 3 M+2 \beta_{k}\left\langle(f-\mu \Phi) x^{*}, x^{k+1}-x^{*}\right\rangle } \\
= & {\left[1-\beta_{k}(\tau-\delta)\right]\left\|x^{k}-x^{*}\right\|^{2}+\beta_{k}(\tau-\delta)\left[\frac{2\left\langle(f-\mu \Phi) x^{*}, x^{k+1}-x^{*}\right\rangle}{\tau-\delta}+\frac{3 M}{\tau-\delta} \frac{\alpha_{k}}{\beta_{k}}\left\|x^{k}-x^{k-1}\right\|\right], }
\end{aligned}
$$

where $\sup _{k \geq 1}\left\{\left\|x^{k}-x^{*}\right\|, \alpha_{k}\left\|x^{k}-x^{k-1}\right\|\right\} \leq M$ for some $M>0$. For each $k \geq 1$, we set

$$
\begin{aligned}
& \Gamma_{k}=\left\|x^{k}-x^{*}\right\|^{2}, \\
& \varepsilon_{k}=\beta_{k}(\tau-\delta), \\
& \vartheta_{k}=\alpha_{k}\left\|x^{k}-x^{k-1}\right\| 3 M+2 \beta_{k}\left\langle(f-\mu \Phi) x^{*}, x^{k+1}-x^{*}\right\rangle .
\end{aligned}
$$

Then (3.12) can be rewritten as

$$
\Gamma_{k+1} \leq\left(1-\varepsilon_{k}\right) \Gamma_{k}+\vartheta_{k} \quad \forall k \geq 1 .
$$

Step 7. Show that the following statements hold:

(a) If $\left\{u^{k_{l}}\right\} \subset\left\{u^{k}\right\}, u^{k_{l}} \rightarrow \bar{u}$ and $t^{k_{l}}-u^{k_{l}} \rightarrow 0$ as $l \rightarrow \infty$, then $\bar{u} \in \operatorname{Sol}(Q, G)$.

(b) If $\left\{\bar{u}^{k_{l}}\right\} \subset\left\{\bar{u}^{k}\right\}, \bar{u}^{k_{l}} \rightarrow \tilde{u}$ and $y^{k_{l}}-\bar{u}^{k_{l}} \rightarrow 0$ as $l \rightarrow \infty$, then $\tilde{u} \in \operatorname{Sol}(C, F)$.

From $\left\{u^{k}\right\} \subset Q$ and $u^{k_{l}} \rightarrow \bar{u}$, we have $\bar{u} \in Q$. From $u^{k_{l}} \rightarrow \bar{u}$, we know that $\left\{u^{k_{l}}\right\}$ is bounded. It follows from (3.4) that $\left\{t^{k_{l}}\right\}$ is bounded. 
Case I. Suppose that there exists a subsequence of $\left\{u^{k_{l}}\right\}$, still denoted by $\left\{u^{k_{l}}\right\}$, such that $t^{k_{l}}=u^{k_{l}}, \forall l \geq 1$. If $v^{k_{l}} \neq u^{k_{l}}$, then from Step 2, we have $\left\|t^{k_{l}}-A x^{*}\right\|<\left\|u^{k_{l}}-A x^{*}\right\|$. This contradicts $t^{k_{l}}=u^{k_{l}}$. Hence, $v^{k_{l}}=u^{k_{l}}$, i.e., $P_{Q}\left(u^{k_{l}}-G u^{k_{l}}\right)=u^{k_{l}} \forall l \geq 1$. Note that

$$
\left\langle G u^{k_{l}}, v-u^{k_{l}}\right\rangle \geq 0, \quad \forall v \in Q .
$$

From $u^{k_{l}} \rightarrow \bar{u}$, we have $G u^{k_{l}} \rightarrow G \bar{u}$ as $l \rightarrow \infty$. Thus, $\langle G \bar{u}, v-\bar{u}\rangle \geq 0, \forall v \in Q$, i.e., $\bar{u} \in \operatorname{Sol}(Q, G)$.

Case II. Suppose that there exists a subsequence of $\left\{u^{k_{l}}\right\}$, still denoted by $\left\{u^{k_{l}}\right\}$, such that $t^{k_{l}} \neq u^{k_{l}}, \forall l \geq 1$. Let $\left\{i_{l}\right\}$ be the sequence of the smallest nonnegative integers such that, for all $l \geq 1$,

$$
\left\langle G\left(\left(1-\gamma^{i_{l}}\right) u^{k_{l}}+\gamma^{i_{l}} v^{k_{l}}\right), u^{k_{l}}-v^{k_{l}}\right\rangle \geq \frac{\left\|u^{k_{l}}-v^{k_{l}}\right\|^{2}}{2},
$$

where $v^{k_{l}}=P_{Q}\left(u^{k_{l}}-G u^{k_{l}}\right), w^{k_{l}}=\left(1-\gamma_{k_{l}}\right) u^{k_{l}}+\gamma_{k_{l}} v^{k_{l}}$ and $\gamma_{k_{l}}=\gamma^{i_{l}}$. According to Step 2 (a), we have

$$
\left\|t^{k_{l}}-A x^{*}\right\|^{2} \leq\left\|u^{k_{l}}-A x^{*}\right\|^{2}-\left(\sigma_{k_{l}}\left\|G w^{k_{l}}\right\|\right)^{2},
$$

where $\sigma_{k_{l}}=\left\langle G w^{k_{l}}, u^{k_{l}}-w^{k_{l}}\right\rangle /\left\|G w^{k_{l}}\right\|^{2}$. Thus,

$$
\begin{aligned}
\left(\sigma_{k_{l}}\left\|G w^{k_{l}}\right\|\right)^{2} & \leq\left\|u^{k_{l}}-A x^{*}\right\|^{2}-\left\|t^{k_{l}}-A x^{*}\right\|^{2} \\
& \leq\left(\left\|u^{k_{l}}-A x^{*}\right\|+\left\|t^{k_{l}}-A x^{*}\right\|\right)\left\|u^{k_{l}}-t^{k_{l}}\right\|,
\end{aligned}
$$

which, together with the boundedness of $\left\{u^{k_{l}}\right\},\left\{t^{k_{l}}\right\}$ and $\left\|t^{k_{l}}-u^{k_{l}}\right\| \rightarrow 0$, implies that

$$
\lim _{l \rightarrow \infty} \sigma_{k_{l}}\left\|G w^{k_{l}}\right\|=0 \text {. }
$$

From $\left\{u^{k_{l}}\right\} \subset Q$, we get

$$
\left\|v^{k_{l}}-u^{k_{l}}\right\|=\left\|P_{Q}\left(u^{k_{l}}-G u^{k_{l}}\right)-P_{Q} u^{k_{l}}\right\| \leq\left\|G u^{k_{l}}\right\|,
$$

which, together with the boundedness of $\left\{u^{k_{l}}\right\}$ and $\left\{G u^{k_{l}}\right\}$, implies that $\left\{v^{k_{l}}\right\}$ is bounded. We also infer that $\left\{w^{k_{l}}\right\}$ is bounded. Thus, $\left\{G w^{k_{l}}\right\}$ is bounded. So, from $\left\langle G w^{k_{l}}, u^{k_{l}}-w^{k_{l}}\right\rangle=$ $\sigma_{k_{l}}\left\|G w^{k_{l}}\right\|^{2}$, we have

$$
\lim _{l \rightarrow \infty}\left\langle G w^{k_{l}}, u^{k_{l}}-w^{k_{l}}\right\rangle=0 .
$$

In view of $w^{k_{l}}=\left(1-\gamma_{k_{l}}\right) u^{k_{l}}+\gamma_{k_{l}} v^{k_{l}}$, we get

$$
\left\langle G w^{k_{l}}, u^{k_{l}}-w^{k_{l}}\right\rangle=\gamma_{k_{l}}\left\langle G w^{k_{l}}, u^{k_{l}}-v^{k_{l}}\right\rangle \geq \frac{\gamma_{k_{l}}\left\|u^{k_{l}}-v^{k_{l}}\right\|^{2}}{2} .
$$

It follows that

$$
\lim _{l \rightarrow \infty} \gamma_{k_{l}}\left\|u^{k_{l}}-v^{k_{l}}\right\|^{2}=0
$$

From $v^{k_{l}}=P_{Q}\left(u^{k_{l}}-G u^{k_{l}}\right)$, we have

$$
\left\langle u^{k_{l}}-G u^{k_{l}}-v^{k_{l}}, v-v^{k_{l}}\right\rangle \leq 0 \quad \forall v \in Q,
$$

which immediately yields

$$
\left\langle G u^{k_{l}}, v-v^{k_{l}}\right\rangle \geq\left\langle u^{k_{l}}-v^{k_{l}}, v-v^{k_{l}}\right\rangle, \quad \forall v \in Q .
$$

We now discuss two distinct cases. 
Case II.1. $\limsup _{l \rightarrow \infty} \gamma_{k_{l}}>0$. In this case, there exist $\bar{\gamma}$ and a subsequence of $\left\{\gamma_{k_{l}}\right\}$, still denoted by $\left\{\gamma_{k_{l}}\right\}$ such that $\gamma_{k_{l}} \rightarrow \bar{\gamma}$. From (3.14), we deduce that $\lim _{l \rightarrow \infty}\left\|u^{k_{l}}-v^{k_{l}}\right\|=0$. From $u^{k_{l}} \rightarrow \bar{u}$, we have $v^{k_{l}} \rightarrow \bar{u}$. Since $u^{k_{l}}-v^{k_{l}} \rightarrow 0$ and $\left\{v^{k_{l}}\right\}$ is bounded, we have

$$
\lim _{l \rightarrow \infty}\left\langle u^{k_{l}}-v^{k_{l}}, v-v^{k_{l}}\right\rangle=0 .
$$

From (3.15), $u^{k_{l}} \rightarrow \bar{u}$ and $v^{k_{l}} \rightarrow \bar{u}$, we get $\langle G \bar{u}, v-\bar{u}\rangle \geq 0, \forall v \in Q$, i.e., $\bar{u} \in \operatorname{Sol}(Q, G)$.

Case II.2. $\lim _{l \rightarrow \infty} \gamma_{k_{l}}=0$. From the boundedness of $\left\{v^{k_{l}}\right\}$, without loss of generality, we may assume that $v^{k_{l}} \rightarrow \bar{v}$ as $l \rightarrow \infty$. From $\gamma^{j_{l}}=\gamma_{k_{l}} \rightarrow 0$ as $l \rightarrow \infty$, we have that $j_{l}>1$ for $l$ enough large, and hence

$$
\left\langle G q^{k_{l}}, u^{k_{l}}-v^{k_{l}}\right\rangle<\frac{\left\|u^{k_{l}}-v^{k_{l}}\right\|^{2}}{2},
$$

where $q^{k_{l}}=\left(1-\gamma^{i_{l}-1}\right) u^{k_{l}}+\gamma^{i_{l}-1} v^{k_{l}}$. Putting $v=u^{k_{l}}$ in (3.15), we have

$$
\left\langle G u^{k_{l}}, u^{k_{l}}-v^{k_{l}}\right\rangle \geq\left\|u^{k_{l}}-v^{k_{l}}\right\|^{2} .
$$

It follows that

$$
\left\langle G q^{k_{l}}, u^{k_{l}}-v^{k_{l}}\right\rangle<\frac{1}{2}\left\langle G u^{k_{l}}, u^{k_{l}}-v^{k_{l}}\right\rangle .
$$

Note that $\left\{u^{k_{l}}\right\}$ and $\left\{v^{k_{l}}\right\}$ are bounded and $\gamma_{k_{l}} \rightarrow 0$. So it follows that

$$
\left\|q^{k_{l}}-u^{k_{l}}\right\|=\frac{\gamma_{k_{l}}}{\gamma}\left\|u^{k_{l}}-v^{k_{l}}\right\| \rightarrow 0 \quad(l \rightarrow \infty) .
$$

From $u^{k_{l}} \rightarrow \bar{u}$ and $q^{k_{l}}-u^{k_{l}} \rightarrow 0$, we know that $q^{k_{l}} \rightarrow \bar{u}$. Since $v^{k_{l}} \rightarrow \bar{v}$, we get from (3.17) that $\langle G \bar{u}, \bar{u}-\bar{v}\rangle \leq \frac{1}{2}\langle G \bar{u}, \bar{u}-\bar{v}\rangle$. Thus,

$$
\langle G \bar{u}, \bar{u}-\bar{v}\rangle \leq 0
$$

Note that

$$
\left\langle G u^{k_{l}}, u^{k_{l}}-v^{k_{l}}\right\rangle \geq 0 .
$$

From $u^{k_{l}} \rightarrow \bar{u}$ and $v^{k_{l}} \rightarrow \bar{v}$, we get $\langle G \bar{u}, \bar{u}-\bar{v}\rangle \geq 0$, which leads to

$$
\langle G \bar{u}, \bar{u}-\bar{v}\rangle=0 .
$$

This further implies that

$$
\lim _{l \rightarrow \infty}\left\langle G u^{k_{l}}, u^{k_{l}}-v^{k_{l}}\right\rangle=\langle G \bar{u}, \bar{u}-\bar{v}\rangle=0 .
$$

Hence, $\lim _{l \rightarrow \infty}\left\|u^{k_{l}}-v^{k_{l}}\right\|=0$, which together with $u^{k_{l}} \rightarrow \bar{u}$, yields $v^{k_{l}} \rightarrow \bar{u}$. From $u^{k_{l}}-v^{k_{l}} \rightarrow 0$ and the boundedness of $\left\{v^{k_{l}}\right\}$, we get $\lim _{l \rightarrow \infty}\left\langle u^{k_{l}}-v^{k_{l}}, v-v^{k_{l}}\right\rangle=0$. So, from (3.15), $u^{k_{l}} \rightarrow \bar{u}$ and $v^{k_{l}} \rightarrow \bar{u}$, we get $\langle G \bar{u}, v-\bar{u}\rangle \geq 0, \forall v \in Q$, i.e., $\bar{u} \in \operatorname{Sol}(Q, G)$. Similarly, we can obtain (b).

Step 8. Show that $\left\{x^{k}\right\}$ converges strongly to the unique solution $x^{*} \in \operatorname{Fix}(T) \cap \Omega$ of the VIP: $\left\langle(\mu \Phi-f) x^{*}, p-x^{*}\right\rangle \geq 0, \forall p \in \operatorname{Fix}(T) \cap \Omega$.

Indeed, it is sufficient to show the convergence of $\left\{\Gamma_{k}\right\}$ to zero by the following two cases:

Case 1. Suppose that there exists a positive integer $k_{0} \geq 1$ such that $\left\{\Gamma_{k}\right\}$ is nonincreasing for $k \geq k_{0}$. Then, we obtain that the $\operatorname{limit}_{k \rightarrow \infty}\left\|x^{k}-x^{*}\right\|$ exists and

$$
\Gamma_{k}-\Gamma_{k+1} \rightarrow 0 \quad(k \rightarrow \infty) .
$$


So, it follows from (3.6), (3.8) and (3.11) that

$$
\begin{aligned}
& \left\|x^{k+1}-x^{*}\right\|^{2} \\
& \leq\left[1-\beta_{k}(\tau-\delta)-v_{k}\right]\left\|\bar{u}^{k}-x^{*}\right\|^{2}+v_{k}\left\|p^{k}-x^{*}\right\|^{2}+2 \beta_{k}\left\langle(f-\mu \Phi) x^{*}, x^{k+1}-x^{*}\right\rangle \\
& \leq\left[1-\beta_{k}(\tau-\delta)-v_{k}\right] \|\left[\left\|p^{k}-x^{*}\right\|^{2}-\delta_{k}\left(1-\delta_{k}\|A\|^{2}\right)\left\|t^{k}-A p^{k}\right\|^{2}-\delta_{k}\left\|u^{k}-A p^{k}\right\|^{2}\right] \\
& +v_{k}\left\|p^{k}-x^{*}\right\|^{2}+2 \beta_{k}\left\|(f-\mu \Phi) x^{*}\right\|\left\|x^{k+1}-x^{*}\right\| \\
& \leq\left[1-\beta_{k}(\tau-\delta)\right]\left\|p^{k}-x^{*}\right\|^{2}-\left[1-\beta_{k}(\tau-\delta)-v_{k}\right]\left[\delta_{k}\left(1-\delta_{k}\|A\|^{2}\right)\left\|t^{k}-A p^{k}\right\|^{2}\right. \\
& \left.+\delta_{k}\left\|u^{k}-A p^{k}\right\|^{2}\right]+\beta_{k} M_{1} \\
& \leq\left\|x^{k}-x^{*}\right\|^{2}+\alpha_{k}\left\|x^{k}-x^{k-1}\right\| 3 M-\left[1-\beta_{k}(\tau-\delta)-v_{k}\right]\left[\delta_{k}\left(1-\delta_{k}\|A\|^{2}\right)\left\|t^{k}-A p^{k}\right\|^{2}\right. \\
& \left.+\delta_{k}\left\|u^{k}-A p^{k}\right\|^{2}\right]+\beta_{k} M_{1},
\end{aligned}
$$

where $\sup _{k \geq 0} 2\left\|(f-\mu \Phi) x^{*}\right\|\left\|x^{k}-x^{*}\right\| \leq M_{1}$ for some $M_{1}>0$. This immediately arrives at

$$
\begin{aligned}
& {\left[1-\beta_{k}(\tau-\delta)-v_{k}\right]\left[\delta_{k}\left(1-\delta_{k}\|A\|^{2}\right)\left\|t^{k}-A p^{k}\right\|^{2}+\delta_{k}\left\|u^{k}-A p^{k}\right\|^{2}\right]} \\
& \leq \Gamma_{k}-\Gamma_{k+1}+\alpha_{k}\left\|x^{k}-x^{k-1}\right\| 3 M+\beta_{k} M_{1} \quad \forall k \geq k_{0} .
\end{aligned}
$$

Since $\left\{\delta_{k}\right\} \subset[\underline{\delta}, \bar{\delta}] \subset\left(0, \frac{1}{1+\|A\|^{2}}\right), \Gamma_{k}-\Gamma_{k+1} \rightarrow 0, \beta_{k} \rightarrow 0, \alpha_{k}\left\|x^{k}-x^{k-1}\right\| \rightarrow 0$ and $\liminf _{k \rightarrow \infty}(1-$ $\left.v_{k}\right)>0$, we get

$$
\lim _{k \rightarrow \infty}\left\|t^{k}-A p^{k}\right\|=0 \quad \text { and } \quad \lim _{k \rightarrow \infty}\left\|u^{k}-A p^{k}\right\|=0
$$

which further yields that

$$
\lim _{k \rightarrow \infty}\left\|t^{k}-u^{k}\right\|=0 \text {. }
$$

In view of $p^{k}-x^{k}=\alpha_{k}\left(x^{k}-x^{k-1}\right)$ and $\alpha_{k}\left\|x^{k}-x^{k-1}\right\| \rightarrow 0$, we get

$$
\lim _{k \rightarrow \infty}\left\|p^{k}-x^{k}\right\|=0 \text {. }
$$

Since the projection operator $P_{C}$ is nonexpansive and $\left\{x^{k}\right\} \subset C$, we can write

$$
\begin{aligned}
\left\|x^{k}-\bar{u}^{k}\right\| & \leq\left\|x^{k}-p^{k}-\delta_{k} A^{*}\left(t^{k}-A p^{k}\right)\right\| \\
& \leq\left\|x^{k}-p^{k}\right\|+\delta_{k}\left\|A^{*}\right\|\left\|t^{k}-A p^{k}\right\| \\
& \leq\left\|x^{k}-p^{k}\right\|+\bar{\delta}\|A\|\left\|t^{k}-A p^{k}\right\|,
\end{aligned}
$$

which, together with (3.19), leads to

$$
\lim _{k \rightarrow \infty}\left\|x^{k}-\bar{u}^{k}\right\|=0
$$

Using (3.7), (3.8) and (3.11) that, for all $k \geq k_{0}$,

$$
\begin{aligned}
& \left\|x^{k+1}-x^{*}\right\|^{2} \\
& \leq\left[1-\beta_{k}(\tau-\delta)-v_{k}\right]\left\|z^{k}-x^{*}\right\|^{2}+v_{k}\left\|p^{k}-x^{*}\right\|^{2}+2 \beta_{k}\left\|(f-\mu \Phi) x^{*}\right\|\left\|x^{k+1}-x^{*}\right\| \\
& \leq\left[1-\beta_{k}(\tau-\delta)-v_{k}\right]\left(\left\|\bar{u}^{k}-x^{*}\right\|^{2}-\lambda_{k}\left(1-\lambda_{k}\right)\left\|y^{k}-\bar{u}^{k}\right\|^{2}\right)+v_{k}\left\|p^{k}-x^{*}\right\|^{2}+\beta_{k} M_{1} \\
& \leq\left[1-\beta_{k}(\tau-\delta)\right]\left\|p^{k}-x^{*}\right\|^{2}-\left[1-\beta_{k}(\tau-\delta)-v_{k}\right] \lambda_{k}\left(1-\lambda_{k}\right)\left\|y^{k}-\bar{u}^{k}\right\|^{2}+\beta_{k} M_{1} \\
& \leq\left\|x^{k}-x^{*}\right\|^{2}+\alpha_{k}\left\|x^{k}-x^{k-1}\right\| 3 M-\left[1-\beta_{k}(\tau-\delta)-v_{k}\right] \lambda_{k}\left(1-\lambda_{k}\right)\left\|y^{k}-\bar{u}^{k}\right\|^{2}+\beta_{k} M_{1} .
\end{aligned}
$$


This immediately leads to

$$
\left[1-\beta_{k}(\tau-\delta)-v_{k}\right] \lambda_{k}\left(1-\lambda_{k}\right)\left\|y^{k}-\bar{u}^{k}\right\|^{2} \leq \Gamma_{k}-\Gamma_{k+1}+\alpha_{k}\left\|x^{k}-x^{k-1}\right\| 3 M+\beta_{k} M_{1}, \quad \forall k \geq k_{0} .
$$

Since $\lambda_{k} \rightarrow \lambda \in(0,1), \Gamma_{k}-\Gamma_{k+1} \rightarrow 0, \beta_{k} \rightarrow 0, \alpha_{k}\left\|x^{k}-x^{k-1}\right\| \rightarrow 0$ and $\liminf _{k \rightarrow \infty}\left(1-v_{k}\right)>0$, we get

$$
\lim _{n \rightarrow \infty}\left\|y^{k}-\bar{u}^{k}\right\|=0
$$

Note that, for all $k \geq 1$,

$$
\left\|z^{k}-\bar{u}^{k}\right\|=\lambda_{k}\left\|y^{k}-\bar{u}^{k}\right\| \leq\left\|y^{k}-\bar{u}^{k}\right\|
$$

which, together with (3.21), implies that

$$
\lim _{k \rightarrow \infty}\left\|z^{k}-\bar{u}^{k}\right\|=0
$$

Taking into account the inequality $\left\|x^{k}-z^{k}\right\| \leq\left\|x^{k}-\bar{u}^{k}\right\|+\left\|\bar{u}^{k}-z^{k}\right\|, \forall k \geq 1$, we find from (3.20) and (3.22) that

$$
\lim _{k \rightarrow \infty}\left\|x^{k}-z^{k}\right\|=0
$$

In view of

$$
x^{k+1}-x^{*}=\beta_{k}\left(f\left(z^{k}\right)-\mu \Phi T z^{k}\right)+v_{k}\left(p^{k}-x^{*}\right)+\left(1-v_{k}\right)\left(T z^{k}-x^{*}\right),
$$

we deduce from (3.8) that

$$
\begin{aligned}
\left\|x^{k+1}-x^{*}\right\|^{2} \leq & \left\|v_{k}\left(p^{k}-x^{*}\right)+\left(1-v_{k}\right)\left(T z^{k}-x^{*}\right)\right\|^{2}+2 \beta_{k}\left\langle f\left(z^{k}\right)-\mu \Phi T z^{k}, x^{k+1}-x^{*}\right\rangle \\
\leq & v_{k}\left\|p^{k}-x^{*}\right\|^{2}+\left(1-v_{k}\right)\left\|T z^{k}-x^{*}\right\|^{2}-v_{k}\left(1-v_{k}\right)\left\|p^{k}-T z^{k}\right\|^{2} \\
& +2 \beta_{k}\left\|f\left(z^{k}\right)-\mu \Phi T z^{k}\right\|\left\|x^{k+1}-x^{*}\right\| \\
\leq & v_{k}\left\|p^{k}-x^{*}\right\|^{2}+\left(1-v_{k}\right)\left\|z^{k}-x^{*}\right\|^{2}-v_{k}\left(1-v_{k}\right)\left\|p^{k}-T z^{k}\right\|^{2} \\
& +2 \beta_{k}\left\|f\left(z^{k}\right)-\mu \Phi T z^{k}\right\|\left\|x^{k+1}-x^{*}\right\| \\
\leq & \left\|p^{k}-x^{*}\right\|^{2}-v_{k}\left(1-v_{k}\right)\left\|p^{k}-T z^{k}\right\|^{2}+2 \beta_{k}\left\|f\left(z^{k}\right)-\mu \Phi T z^{k}\right\|\left\|x^{k+1}-x^{*}\right\| \\
\leq & \left\|x^{k}-x^{*}\right\|^{2}+\alpha_{k}\left\|x^{k}-x^{k-1}\right\| 3 M-v_{k}\left(1-v_{k}\right)\left\|p^{k}-T z^{k}\right\|^{2} \\
& +2 \beta_{k}\left\|f\left(z^{k}\right)-\mu \Phi T z^{k}\right\|\left\|x^{k+1}-x^{*}\right\|,
\end{aligned}
$$

which further yields

$$
v_{k}\left(1-v_{k}\right)\left\|p^{k}-T z^{k}\right\|^{2} \leq \Gamma_{k}-\Gamma_{k+1}+\alpha_{k}\left\|x^{k}-x^{k-1}\right\| 3 M+2 \beta_{k}\left\|f\left(z^{k}\right)-\mu \Phi T z^{k}\right\|\left\|x^{k+1}-x^{*}\right\| .
$$

Since $\beta_{k} \rightarrow 0, \Gamma_{k}-\Gamma_{k+1} \rightarrow 0, \alpha_{k}\left\|x^{k}-x^{k-1}\right\| \rightarrow 0$ and $0<\liminf _{k \rightarrow \infty} v_{k} \leq \limsup _{k \rightarrow \infty} v_{k}<1$, we have from the boundedness of the sequences $\left\{x^{k}\right\}$ and $\left\{z^{k}\right\}$ that $\lim _{k \rightarrow \infty}\left\|p^{k}-T z^{k}\right\|=0$. Thus,

$$
\begin{aligned}
\left\|x^{k+1}-p^{k}\right\| & \leq\left\|\beta_{k}\left(f\left(z^{k}\right)-\mu \Phi T z^{k}\right)+\left(1-v_{k}\right)\left(T z^{k}-p^{k}\right)\right\| \\
& \leq \beta_{k}\left\|f\left(z^{k}\right)-\mu \Phi T z^{k}\right\|+\left\|T z^{k}-p^{k}\right\| \rightarrow 0 \quad(k \rightarrow \infty) .
\end{aligned}
$$

This together with (3.19) implies that

$$
\left\|x^{k+1}-x^{k}\right\| \leq\left\|x^{k+1}-p^{k}\right\|+\left\|p^{k}-x^{k}\right\| \rightarrow 0 \quad(k \rightarrow \infty) .
$$

From the fact that

$$
\left\|z^{k}-T z^{k}\right\| \leq\left\|z^{k}-x^{k}\right\|+\left\|x^{k}-p^{k}\right\|+\left\|p^{k}-T z^{k}\right\|,
$$

we conclude from (3.19) and (3.23) that $\lim _{k \rightarrow \infty}\left\|z^{k}-T z^{k}\right\|=0$. 
Now, we claim that

$$
\limsup _{k \rightarrow \infty}\left\langle(f-\mu \Phi) x^{*}, x^{k+1}-x^{*}\right\rangle \leq 0 .
$$

In fact, from the boundedness of $\left\{x^{k}\right\}$, it follows that there exists a subsequence $\left\{x^{k_{l}}\right\}$ of $\left\{x^{k}\right\}$ such that

$$
\limsup _{k \rightarrow \infty}\left\langle(f-\mu \Phi) x^{*}, x^{k}-x^{*}\right\rangle=\lim _{l \rightarrow \infty}\left\langle(f-\mu \Phi) x^{*}, x^{k_{l}}-x^{*}\right\rangle .
$$

Since $\mathscr{H}_{1}$ is reflexive and $\left\{x^{k}\right\}$ is bounded, we may assume, without loss of generality, that $x^{k_{l}} \rightarrow \tilde{x} \in C$. Hence

$$
\limsup _{k \rightarrow \infty}\left\langle(f-\mu \Phi) x^{*}, x^{k}-x^{*}\right\rangle=\lim _{l \rightarrow \infty}\left\langle(f-\mu \Phi) x^{*}, x^{k_{l}}-x^{*}\right\rangle=\left\langle(f-\mu \Phi) x^{*}, \tilde{x}-x^{*}\right\rangle .
$$

From (3.22), (3.23) and $x^{k_{l}} \rightarrow \tilde{x}$, we deduce that $\bar{u}^{k_{l}} \rightarrow \tilde{x}$ and $z^{k_{l}} \rightarrow \tilde{x}$. From (3.21), it follows that $\lim _{l \rightarrow \infty}\left\|y^{k_{l}}-\bar{u}^{k_{l}}\right\|=0$. So, utilizing $\bar{u}^{k_{l}} \rightarrow \tilde{x}$ and Step 7 (b), we have $\tilde{x} \in \operatorname{Sol}(C, F)$. From $p^{k_{l}} \rightarrow \tilde{x}$, we have $A p^{k_{l}} \rightarrow A \tilde{x}$. This implies that $u^{k_{l}} \rightarrow A \tilde{x}$. From (3.18), we obtain $\lim _{l \rightarrow \infty} \| t^{k_{l}}-$ $u^{k_{l}} \|=0$. Hence, utilizing $u^{k_{l}} \rightarrow A \tilde{x}$ and Step 7 (a), we have $A \tilde{x} \in \operatorname{Sol}(Q, G)$. According to $\tilde{x} \in \operatorname{Sol}(C, F)$ and $A \tilde{x} \in \operatorname{Sol}(Q, G)$, we have $\tilde{x} \in \Omega$. Note that $\lim _{k \rightarrow \infty}\left\|z^{k}-T z^{k}\right\|=0$. From the demiclosedness of $I-T$ at zero, we deduce from $z^{k_{l}} \rightarrow \tilde{x}$ that $\tilde{x} \in \operatorname{Fix}(T)$. Consequently, $\tilde{x} \in \operatorname{Fix}(T) \cap \Omega$. Hence from (3.2) and (3.25), we get

$$
\limsup _{k \rightarrow \infty}\left\langle(f-\mu \Phi) x^{*}, x^{k}-x^{*}\right\rangle=\left\langle(f-\mu \Phi) x^{*}, \tilde{x}-x^{*}\right\rangle \leq 0
$$

which leads to

$$
\begin{aligned}
& \limsup _{k \rightarrow \infty}\left\langle(f-\mu \Phi) x^{*}, x^{k+1}-x^{*}\right\rangle \\
& =\limsup _{k \rightarrow \infty}\left[\left\langle(f-\mu \Phi) x^{*}, x^{k+1}-x^{k}\right\rangle+\left\langle(f-\mu \Phi) x^{*}, x^{k}-x^{*}\right\rangle\right] \\
& \leq \limsup _{k \rightarrow \infty}\left[\left\|(f-\mu \Phi) x^{*}\right\|\left\|x^{k+1}-x^{k}\right\|+\left\langle(f-\mu \Phi) x^{*}, x^{k}-x^{*}\right\rangle\right] \leq 0 .
\end{aligned}
$$

Note that $\left\{\beta_{k}(\tau-\delta)\right\} \subset[0,1], \sum_{k=1}^{\infty} \beta_{k}(\tau-\delta)=\infty$, and

$$
\limsup _{k \rightarrow \infty}\left[\frac{2\left\langle(f-\mu \Phi) x^{*}, x^{k+1}-x^{*}\right\rangle}{\tau-\delta}+\frac{3 M}{\tau-\delta} \cdot \frac{\alpha_{k}}{\beta_{k}}\left\|x^{k}-x^{k-1}\right\|\right] \leq 0 .
$$

Therefore, applying Lemma 2.2 to (3.12), we get $\lim _{k \rightarrow \infty}\left\|x_{k}-x^{*}\right\|=0$.

Case 2. Suppose that $\exists\left\{\Gamma_{k_{l}}\right\} \subset\left\{\Gamma_{k}\right\}$ s.t. $\Gamma_{k_{l}}<\Gamma_{k_{l}+1} \forall l \in \mathbf{N}$, where $\mathbf{N}$ is the set of all positive integers. Define the mapping $\tau: \mathbf{N} \rightarrow \mathbf{N}$ by

$$
\tau(k):=\max \left\{l \leq k: \Gamma_{l}<\Gamma_{l+1}\right\} .
$$

Using Lemma 2.5, we have

$$
\Gamma_{\tau(k)} \leq \Gamma_{\tau(k)+1} \quad \text { and } \quad \Gamma_{k} \leq \Gamma_{\tau(k)+1}
$$

Setting $\Gamma_{k}=\left\|x^{k}-x^{*}\right\|^{2}$, we can similarly obtain

$$
\lim _{k \rightarrow \infty}\left\|x^{\tau(k)+1}-x^{\tau(k)}\right\|=0
$$

and

$$
\limsup _{k \rightarrow \infty}\left\langle(f-\mu \Phi) x^{*}, x^{\tau(k)+1}-x^{*}\right\rangle \leq 0 .
$$


Thanks to $\Gamma_{\tau(k)} \leq \Gamma_{\tau(k)+1}$ and $0<\beta_{\tau(k)}$, we conclude from (3.12) that

$$
\left\|x^{\tau(k)}-x^{*}\right\|^{2} \leq \frac{2}{\tau-\delta}\left\langle(f-\mu \Phi) x^{*}, x^{\tau(k)+1}-x^{*}\right\rangle+\frac{3 M}{\tau-\delta} \cdot \frac{\alpha_{\tau(k)}}{\beta_{\tau(k)}}\left\|x^{\tau(k)}-x^{\tau(k)-1}\right\| .
$$

Hence $\limsup _{k \rightarrow \infty}\left\|x^{\tau(k)}-x^{*}\right\|^{2} \leq 0$ and $\lim _{k \rightarrow \infty}\left\|x^{\tau(k)}-x^{*}\right\|^{2}=0$. From (3.27), we have

$$
\begin{aligned}
& \left\|x^{\tau(k)+1}-x^{*}\right\|^{2}-\left\|x^{\tau(k)}-x^{*}\right\|^{2} \\
& =2\left\langle x^{\tau(k)+1}-x^{\tau(k)}, x^{\tau(k)}-x^{*}\right\rangle+\left\|x^{\tau(k)+1}-x^{\tau(k)}\right\|^{2} \\
& \leq 2\left\|x^{\tau(k)+1}-x^{\tau(k)}\right\|\left\|x^{\tau(k)}-x^{*}\right\|+\left\|x^{\tau(k)+1}-x^{\tau(k)}\right\|^{2} \rightarrow 0 \quad(n \rightarrow \infty) .
\end{aligned}
$$

From $\Gamma_{k} \leq \Gamma_{\tau(k)+1}$, we have

$$
\begin{aligned}
\left\|x^{k}-x^{*}\right\|^{2} & \leq\left\|x^{\tau(k)+1}-x^{*}\right\|^{2} \\
& \leq\left\|x^{\tau(k)}-x^{*}\right\|^{2}+2\left\|x^{\tau(k)+1}-x^{\tau(k)}\right\|\left\|x^{\tau(k)}-x^{*}\right\|+\left\|x^{\tau(k)+1}-x^{\tau(k)}\right\|^{2} .
\end{aligned}
$$

It is easy to see from (3.27) that $x^{k} \rightarrow x^{*}$ as $k \rightarrow \infty$. This completes the proof.

If $F=G=0$, we derive the following result from Algorithm 3.1 and Theorem 3.1.

Corollary 3.1. Let $C$ and $Q$ be two closed convex nonempty subsets of two real Hilbert spaces $\mathscr{H}_{1}$ and $\mathscr{H}_{2}$, respectively. Let $f: C \rightarrow \mathscr{H}_{1}$ be a $\delta$-contractive mapping and let $\Phi: C \rightarrow \mathscr{H}_{1}$ be a $\kappa$-Lipschitzian, $\eta$-strongly monotone mapping, where $\delta<\tau:=1-\sqrt{1-\mu\left(2 \eta-\mu \kappa^{2}\right)}$ for $\mu \in\left(0, \frac{2 \eta}{\kappa^{2}}\right)$. Let $T$ be a quasi-nonexpansive self-mapping on $C$ with the fact that $I-T$ is demiclosed at zero such that $\operatorname{Fix}(T) \cap \Gamma \neq \emptyset$ where $\Gamma=\left\{x^{*} \in C: A x^{*} \in Q\right\}$ is the solution set of the SFP. Assume that $\left\{\varepsilon_{k}\right\} \subset(0,1]$ and $\left\{\beta_{k}\right\},\left\{v_{k}\right\} \subset(0,1)$ satisfy

(i) $\beta_{k}+v_{k} \leq 1, \sum_{k=1}^{\infty} \beta_{k}=\infty, \lim _{k \rightarrow \infty} \beta_{k}=0$;

(ii) $\varepsilon_{k}=\circ\left(\beta_{k}\right)$, i.e., $\lim _{k \rightarrow \infty} \frac{\varepsilon_{k}}{\beta_{k}}=0$;

(iii) $0<\liminf _{k \rightarrow \infty} v_{k} \leq \limsup _{k \rightarrow \infty} v_{k}<1$.

For any given $x^{0}, x^{1} \in C, \alpha>0$ and $\left\{\delta_{k}\right\} \subset[\underline{\delta}, \bar{\delta}] \subset\left(0, \frac{1}{1+\|A\|^{2}}\right)$, let the sequence $\left\{x^{k}\right\}$ be constructed as follows:

Inertial Step: Given the iterates $x^{k-1}$ and $x^{k}(k \geq 1)$, calculate $p^{k}=x^{k}+\alpha_{k}\left(x^{k}-x^{k-1}\right)$, where $\alpha_{k}$ is chosen such that $0 \leq \alpha_{k} \leq \bar{\alpha}_{k}$ and

$$
\bar{\alpha}_{k}= \begin{cases}\min \left\{\alpha, \frac{\varepsilon_{k}}{\left\|x^{k}-x^{k-1}\right\|}\right\}, & \text { if } x^{k}-x^{k-1} \neq 0 \\ \alpha, & \text { otherwise. }\end{cases}
$$

Iteration Steps: Compute $x^{k+1}$ below:

$$
\left\{\begin{array}{l}
u^{k}=P_{Q}\left(A p^{k}\right), \\
\bar{u}^{k}=P_{C}\left(p^{k}+\delta_{k} A^{*}\left(u^{k}-A p^{k}\right)\right), \\
x^{k+1}=P_{C}\left[\beta_{k} f\left(\bar{u}^{k}\right)+v_{k} p^{k}+\left(\left(1-v_{k}\right) I-\beta_{k} \mu \Phi\right) T \bar{u}^{k}\right] .
\end{array}\right.
$$

Then $x^{k} \rightarrow x^{*} \in \operatorname{Fix}(T) \cap \Gamma$, which is the unique solution to the VIP: $\left\langle(\mu \Phi-f) x^{*}, p-x^{*}\right\rangle \geq 0, \forall p \in$ $\operatorname{Fix}(T) \cap \Gamma$.

Next, we introduce the other modified inertial linesearch extragradient algorithm. 
Algorithm 3.2. Initial Step: Give $x^{0}, x^{1} \in C$ arbitrarily. Let $\alpha>0, \ell \in(0,1), \gamma \in(0,1)$, $\left\{\boldsymbol{\delta}_{k}\right\} \subset[\underline{\boldsymbol{\delta}}, \overline{\boldsymbol{\delta}}] \subset\left(0, \frac{1}{1+\|A\|^{2}}\right),\left\{\lambda_{k}\right\} \subset(0,1), \lim _{k \rightarrow \infty} \lambda_{k}=\lambda \in(0,1)$.

Iteration Steps: Compute $x^{k+1}$ below:

Step 1. Given the iterates $x^{k-1}$ and $x^{k}(k \geq 1)$, choose $\alpha_{k}$ s.t. $0 \leq \alpha_{k} \leq \bar{\alpha}_{k}$, where

$$
\bar{\alpha}_{k}= \begin{cases}\min \left\{\alpha, \frac{\varepsilon_{k}}{\left\|x^{k}-x^{k-1}\right\|}\right\}, & \text { if } x^{k}-x^{k-1} \neq 0, \\ \alpha, & \text { otherwise. }\end{cases}
$$

Step 2. Set $p^{k}=x^{k}+\alpha_{k}\left(x^{k}-x^{k-1}\right)$ and calculate $u^{k}=P_{Q}\left(A p^{k}\right)$ and $v^{k}=P_{Q}\left(u^{k}-G u^{k}\right)$. If $v^{k}=u^{k}$, then set $t^{k}=u^{k}$ and go to Step 5. Otherwise, go to Step 3 .

Step 3. Find $i_{k}$ as the smallest nonnegative integer $i$ such that $w^{k, i}=\left(1-\gamma^{i}\right) u^{k}+\gamma^{i} v^{k}$ and $\left\langle G w^{k, i}, u^{k}-v^{k}\right\rangle \geq \frac{1}{2}\left\|u^{k}-v^{k}\right\|^{2}$. Set $\gamma_{k}=\gamma^{i_{k}}$ and $w^{k}=w^{k, i_{k}}$.

Step 4. Calculate $t^{k}=P_{Q}\left(u^{k}-\sigma_{k} G w^{k}\right)$, where $\sigma_{k}=\frac{\left\langle G w^{k}, u^{k}-w^{k}\right\rangle}{\left\|G w^{k}\right\|^{2}}$.

Step 5. Calculate $\bar{u}^{k}=P_{C}\left(p^{k}+\delta_{k} A^{*}\left(t^{k}-A p^{k}\right)\right)$ and $\bar{v}^{k}=P_{C}\left(\bar{u}^{k}-F \bar{u}^{k}\right)$. If $\bar{v}^{k}=\bar{u}^{k}$, then set $y^{k}=\bar{u}^{k}$ and go to Step 8. Otherwise, go to Step 6 .

Step 6. Find $j_{k}$ as the smallest nonnegative integer $j$ such that $\bar{w}^{k, j}=\left(1-\ell^{j}\right) \bar{u}^{k}+\ell^{j} \bar{v}^{k}$ and $\left\langle F \bar{w}^{k, j}, \bar{u}^{k}-\bar{v}^{k}\right\rangle \geq \frac{1}{2}\left\|\bar{u}^{k}-\bar{v}^{k}\right\|^{2}$. Set $\ell_{k}=\ell^{j_{k}}$ and $\bar{w}^{k}=\bar{w}^{k, j_{k}}$.

Step 7. Calculate $y^{k}=P_{C}\left(\bar{u}^{k}-\bar{\sigma}_{k} F \bar{w}^{k}\right)$, where $\bar{\sigma}_{k}=\frac{\left\langle F \bar{w}^{k} \bar{u}^{k}-\bar{w}^{k}\right\rangle}{\left\|F \bar{w}^{k}\right\|^{2}}$.

Step 8. Calculate $z^{k}=\left(1-\lambda_{k}\right) \bar{u}^{k}+\lambda_{k} y^{k}$ and

$$
x^{k+1}=P_{C}\left[\beta_{k} f\left(x^{k}\right)+v_{k} x^{k}+\left(\left(1-v_{k}\right) I-\beta_{k} \mu \Phi\right) T z^{k}\right] .
$$

Set $k:=k+1$ and go to Step 1 .

Theorem 3.2. Assume that $\left\{x^{k}\right\}$ is the sequence constructed by Algorithm 3.2. Then $x^{k} \rightarrow x^{*} \in$ $\operatorname{Fix}(T) \cap \Omega$, which is a unique solution to the VIP: $\left\langle(\mu \Phi-f) x^{*}, p-x^{*}\right\rangle \geq 0, \forall p \in \operatorname{Fix}(T) \cap \Omega$.

Proof. As in Theorem 3.1, we deduce that there exists a unique solution $x^{*} \in \operatorname{Fix}(T) \cap \Omega$ to the VIP (3.2). In order to show $\lim _{k \rightarrow \infty}\left\|x^{k}-x^{*}\right\|=0$, we divide the rest of the proof into two steps.

Step 1. Show that $\left\{x^{k}\right\}$ is bounded.

Indeed, from Steps 1-5 in the proof of Theorem 3.1, $\beta_{k}+v_{k} \leq 1$, we obtain from Lemma 2.4 that

$$
\begin{aligned}
\left\|x^{k+1}-x^{*}\right\| \leq & \beta_{k}\left(\left\|f\left(x^{k}\right)-f\left(x^{*}\right)\right\|+\left\|f\left(x^{*}\right)-x^{*}\right\|\right)+v_{k}\left\|x^{k}-x^{*}\right\| \\
& +\left(1-\beta_{k}-v_{k}\right)\left\|\left(\frac{1-v_{k}}{1-\beta_{k}-v_{k}} I-\frac{\beta_{k}}{1-\beta_{k}-v_{k}} \mu \Phi\right) T z^{k}-x^{*}\right\| \\
\leq & \beta_{k}\left(\delta\left\|x^{k}-x^{*}\right\|+\left\|f\left(x^{*}\right)-x^{*}\right\|\right)+v_{k}\left\|x^{k}-x^{*}\right\| \\
& +\left(1-v_{k}\right)\left\|\left(I-\frac{\beta_{k}}{1-v_{k}} \mu \Phi\right) T z^{k}-\left(I-\frac{\beta_{k}}{1-v_{k}} \mu \Phi\right) x^{*}+\frac{\beta_{k}}{1-v_{k}}(I-\mu \Phi) x^{*}\right\| \\
\leq & \beta_{k}\left(\delta\left\|x^{k}-x^{*}\right\|+\left\|f\left(x^{*}\right)-x^{*}\right\|\right)+v_{k}\left\|x^{k}-x^{*}\right\| \\
& +\left(1-v_{k}\right)\left[\left(1-\frac{\beta_{k}}{1-v_{k}} \tau\right)\left\|z^{k}-x^{*}\right\|+\frac{\beta_{k}}{1-v_{k}}\left\|(I-\mu \Phi) x^{*}\right\|\right] \\
\leq & \max \left\{\left\|x^{k}-x^{*}\right\|, \frac{M_{0}+\left\|f\left(x^{*}\right)-x^{*}\right\|+\left\|(I-\mu \Phi) x^{*}\right\|}{\tau-\delta}\right\} .
\end{aligned}
$$


By induction, we obtain $\left\|x^{k}-x^{*}\right\| \leq \max \left\{\left\|x^{1}-x^{*}\right\|, \frac{M_{0}+\left\|f\left(x^{*}\right)-x^{*}\right\|+\left\|(I-\mu \Phi) x^{*}\right\|}{\tau-\delta}\right\} \forall k \geq 1$. Thus, $\left\{x^{k}\right\}$ is bounded, so $\left\{p^{k}\right\},\left\{\bar{u}^{k}\right\},\left\{z^{k}\right\},\left\{f\left(x^{k}\right)\right\},\left\{T z^{k}\right\}$ and $\left\{\Phi T z^{k}\right\}$.

Step 2. Show that $\left\{x^{n}\right\}$ converges strongly to $x^{*}$.

Indeed, putting $\omega^{k}:=\beta_{k} f\left(x^{k}\right)+v_{k} x^{k}+\left(\left(1-v_{k}\right) I-\beta_{k} \mu \Phi\right) T z^{k}$, we have $x^{k+1}=P_{C} \omega^{k}$. It follows that

$$
\begin{aligned}
\left\|x^{k+1}-x^{*}\right\|^{2} \leq & \left\langle\beta_{k}\left(f\left(x^{k}\right)-f\left(x^{*}\right)\right)+v_{k}\left(x^{k}-x^{*}\right)+\left(1-v_{k}\right)\left[\left(I-\frac{\beta_{k}}{1-v_{k}} \mu \Phi\right) T z^{k}\right.\right. \\
& \left.\left.-\left(I-\frac{\beta_{k}}{1-v_{k}} \mu \Phi\right) x^{*}\right], x^{k+1}-x^{*}\right\rangle+\beta_{k}\left\langle(f-\mu \Phi) x^{*}, x^{k+1}-x^{*}\right\rangle \\
\leq & \| \beta_{k}\left(f\left(x^{k}\right)-f\left(x^{*}\right)\right)+v_{k}\left(x^{k}-x^{*}\right)+\left(1-v_{k}\right)\left[\left(I-\frac{\beta_{k}}{1-v_{k}} \mu \Phi\right) T z^{k}\right. \\
& \left.-\left(I-\frac{\beta_{k}}{1-v_{k}} \mu \Phi\right) x^{*}\right]\|\| x^{k+1}-x^{*} \|+\beta_{k}\left\langle(f-\mu \Phi) x^{*}, x^{k+1}-x^{*}\right\rangle \\
\leq & \frac{1}{2}\left\{\| \beta_{k}\left(f\left(x^{k}\right)-f\left(x^{*}\right)\right)+v_{k}\left(x^{k}-x^{*}\right)+\left(1-v_{k}\right)\left[\left(I-\frac{\beta_{k}}{1-v_{k}} \mu \Phi\right) T z^{k}\right.\right. \\
& \left.\left.-\left(I-\frac{\beta_{k}}{1-v_{k}} \mu \Phi\right) x^{*}\right]\left\|^{2}+\right\| x^{k+1}-x^{*} \|^{2}\right\}+\beta_{k}\left\langle(f-\mu \Phi) x^{*}, x^{k+1}-x^{*}\right\rangle .
\end{aligned}
$$

Utilizing Lemma 2.4, we obtain that

$$
\begin{aligned}
&\left\|x^{k+1}-x^{*}\right\|^{2} \\
& \leq {\left[\beta_{k} \delta\left\|x^{k}-x^{*}\right\|+v_{k}\left\|x^{k}-x^{*}\right\|+\left(1-\beta_{k} \tau-v_{k}\right)\left\|z^{k}-x^{*}\right\|\right]^{2} } \\
&+2 \beta_{k}\left\langle(f-\mu \Phi) x^{*}, x^{k+1}-x^{*}\right\rangle \\
& \leq \beta_{k} \delta\left\|x^{k}-x^{*}\right\|^{2}+v_{k}\left\|x^{k}-x^{*}\right\|^{2}+\left(1-\beta_{k} \tau-v_{k}\right)\left\|z^{k}-x^{*}\right\|^{2} \\
&+2 \beta_{k}\left\langle(f-\mu \Phi) x^{*}, x^{k+1}-x^{*}\right\rangle \\
& \leq\left(\beta_{k} \delta+v_{k}\right)\left\|x^{k}-x^{*}\right\|^{2}+\left(1-\beta_{k} \tau-v_{k}\right)\left\|p^{k}-x^{*}\right\|^{2}+2 \beta_{k}\left\langle(f-\mu \Phi) x^{*}, x^{k+1}-x^{*}\right\rangle \\
& \leq\left(\beta_{k} \delta+v_{k}\right)\left\{\left\|x^{k}-x^{*}\right\|^{2}+\alpha_{k}\left\|x^{k}-x^{k-1}\right\|\left[2\left\|x^{k}-x^{*}\right\|+\alpha_{k}\left\|x^{k}-x^{k-1}\right\|\right]\right\} \\
&+\left(1-\beta_{k} \tau-v_{k}\right)\left\{\left\|x^{k}-x^{*}\right\|^{2}+\alpha_{k}\left\|x^{k}-x^{k-1}\right\|\left[2\left\|x^{k}-x^{*}\right\|+\alpha_{k}\left\|x^{k}-x^{k-1}\right\|\right]\right\} \\
&+2 \beta_{k}\left\langle(f-\mu \Phi) x^{*}, x^{k+1}-x^{*}\right\rangle \\
& \leq {\left[1-\beta_{k}(\tau-\delta)\right]\left\|x^{k}-x^{*}\right\|^{2}+\alpha_{k}\left\|x^{k}-x^{k-1}\right\| 3 M+2 \beta_{k}\left\langle(f-\mu \Phi) x^{*}, x^{k+1}-x^{*}\right\rangle } \\
&= {\left[1-\beta_{k}(\tau-\delta)\right]\left\|x^{k}-x^{*}\right\|^{2}+\beta_{k}(\tau-\delta)\left[\frac{2\left\langle(f-\mu \Phi) x^{*}, x^{k+1}-x^{*}\right\rangle}{\tau-\delta}+\frac{3 M}{\tau-\delta} \frac{\alpha_{k}}{\beta_{k}}\left\|x^{k}-x^{k-1}\right\|\right] . }
\end{aligned}
$$

We rewrite it as follows:

$$
\Gamma_{k+1} \leq\left(1-\varepsilon_{k}\right) \Gamma_{k}+\vartheta_{k}, \quad \forall k \geq 1 .
$$

From Step 7 of the proof of Theorem 3.1, we can show that the following statements hold:

(a) If $\left\{u^{k_{l}}\right\} \subset\left\{u^{k}\right\}, u^{k_{l}} \rightarrow \bar{u}$ and $t^{k_{l}}-u^{k_{l}} \rightarrow 0$ as $l \rightarrow \infty$, then $\bar{u} \in \operatorname{Sol}(Q, G)$.

(b) If $\left\{\bar{u}^{k_{l}}\right\} \subset\left\{\bar{u}^{k}\right\}, \bar{u}^{k_{l}} \rightarrow \tilde{u}$ and $y^{k_{l}}-\bar{u}^{k_{l}} \rightarrow 0$ as $l \rightarrow \infty$, then $\tilde{u} \in \operatorname{Sol}(C, F)$.

Finally, we show the convergence of $\left\{\Gamma_{k}\right\}$ to zero by the following two cases. 
Case 1. Suppose that there exists an integer $k_{0} \geq 1$ such that $\left\{\Gamma_{k}\right\}$ is non-increasing for $k \geq k_{0}$. Then we obtain that the $\operatorname{limit}_{k \rightarrow \infty}\left\|x^{k}-x^{*}\right\|$ exists and

$$
\Gamma_{k}-\Gamma_{k+1} \rightarrow 0 \quad(k \rightarrow \infty) .
$$

It follows from (3.6) and (3.8) that, for all $k \geq k_{0}$,

$$
\begin{aligned}
&\left\|x^{k+1}-x^{*}\right\|^{2} \\
& \leq\left(\beta_{k} \delta+v_{k}\right)\left\|x^{k}-x^{*}\right\|^{2}+\left(1-\beta_{k} \tau-v_{k}\right)\left[\left\|p^{k}-x^{*}\right\|^{2}-\delta_{k}\left(1-\delta_{k}\|A\|^{2}\right)\left\|t^{k}-A p^{k}\right\|^{2}\right. \\
&\left.-\delta_{k}\left\|u^{k}-A p^{k}\right\|^{2}\right]+2 \beta_{k}\left\langle(f-\mu \Phi) x^{*}, x^{n+1}-x^{*}\right\rangle \\
& \leq\left(\beta_{k} \delta+v_{k}\right)\left[\left\|x^{k}-x^{*}\right\|^{2}+\alpha_{k}\left\|x^{n}-x^{n-1}\right\| 3 M\right]+\left(1-\beta_{k} \tau-v_{k}\right)\left[\left\|x^{k}-x^{*}\right\|^{2}\right. \\
&\left.+\alpha_{k}\left\|x^{k}-x^{k-1}\right\| 3 M-\delta_{k}\left(1-\delta_{k}\|A\|^{2}\right)\left\|t^{k}-A p^{k}\right\|^{2}-\delta_{k}\left\|u^{k}-A p^{k}\right\|^{2}\right] \\
&+2 \beta_{k}\left\langle(f-\mu \Phi) x^{*}, x^{n+1}-x^{*}\right\rangle \\
&= {\left[1-\beta_{k}(\tau-\delta)\right]\left[\left\|x^{k}-x^{*}\right\|^{2}+\alpha_{k}\left\|x^{k}-x^{k-1}\right\| 3 M\right]-\left(1-\beta_{k} \tau-v_{k}\right)\left[\delta_{k}\left(1-\delta_{k}\|A\|^{2}\right)\right.} \\
&\left.\times\left\|t^{k}-A p^{k}\right\|^{2}+\delta_{k}\left\|u^{k}-A p^{k}\right\|^{2}\right]+2 \beta_{k}\left\langle(f-\mu \Phi) x^{*}, x^{k+1}-x^{*}\right\rangle \\
& \leq\left\|x^{k}-x^{*}\right\|^{2}+\alpha_{k}\left\|x^{k}-x^{k-1}\right\| 3 M-\left(1-\beta_{k} \tau-v_{k}\right)\left[\delta_{k}\left(1-\delta_{k}\|A\|^{2}\right)\left\|t^{k}-A p^{k}\right\|^{2}\right. \\
&\left.+\delta_{k}\left\|u^{k}-A p^{k}\right\|^{2}\right]+\beta_{k} M_{1} .
\end{aligned}
$$

This immediately yields

$$
\begin{aligned}
& \left(1-\beta_{k} \tau-v_{k}\right)\left[\delta_{k}\left(1-\delta_{k}\|A\|^{2}\right)\left\|t^{k}-A p^{k}\right\|^{2}+\delta_{k}\left\|u^{k}-A p^{k}\right\|^{2}\right] \\
& \leq \Gamma_{k}-\Gamma_{k+1}+\alpha_{k}\left\|x^{k}-x^{k-1}\right\| 3 M+\beta_{k} M_{1}, \quad \forall k \geq k_{0} .
\end{aligned}
$$

Since $\left\{\boldsymbol{\delta}_{k}\right\} \subset[\underline{\boldsymbol{\delta}}, \overline{\boldsymbol{\delta}}] \subset\left(0, \frac{1}{1+\|A\|^{2}}\right), \Gamma_{k}-\Gamma_{k+1} \rightarrow 0, \beta_{k} \rightarrow 0, \alpha_{k}\left\|x^{k}-x^{k-1}\right\| \rightarrow 0$ and $\liminf _{k \rightarrow \infty}(1-$ $\left.v_{k}\right)>0$, we get

$$
\lim _{k \rightarrow \infty}\left\|t^{k}-A p^{k}\right\|=0 \quad \text { and } \quad \lim _{k \rightarrow \infty}\left\|u^{k}-A p^{k}\right\|=0 .
$$

Consequently, $\lim _{k \rightarrow \infty}\left\|t^{k}-u^{k}\right\|=0$. As in Theorem 3.1, we can obtain that

$$
\lim _{k \rightarrow \infty}\left\|p^{k}-x^{k}\right\|=\lim _{k \rightarrow \infty}\left\|x^{k}-\bar{u}^{k}\right\|=\lim _{k \rightarrow \infty}\left\|y^{k}-\bar{u}^{k}\right\|=\lim _{k \rightarrow \infty}\left\|z^{k}-\bar{u}^{k}\right\|=\lim _{k \rightarrow \infty}\left\|x^{k}-z^{k}\right\|=0 .
$$

By use of (3.8), we deduce that

$$
\begin{aligned}
\left\|x^{k+1}-x^{*}\right\|^{2} \leq & \left\|v_{k}\left(x^{k}-x^{*}\right)+\left(1-v_{k}\right)\left(T z^{k}-x^{*}\right)\right\|^{2}+2 \beta_{k}\left\langle f\left(x^{k}\right)-\mu \Phi T z^{k}, x^{k+1}-x^{*}\right\rangle \\
\leq & v_{k}\left\|x^{k}-x^{*}\right\|^{2}+\left(1-v_{k}\right)\left\|T z^{k}-x^{*}\right\|^{2}-v_{k}\left(1-v_{k}\right)\left\|x^{k}-T z^{k}\right\|^{2} \\
& +2 \beta_{k}\left\|f\left(x^{k}\right)-\mu \Phi T z^{k}\right\|\left\|x^{k+1}-x^{*}\right\| \\
\leq & v_{k}\left\|x^{k}-x^{*}\right\|^{2}+\left(1-v_{k}\right)\left\|p^{k}-x^{*}\right\|^{2}-v_{k}\left(1-v_{k}\right)\left\|x^{k}-T z^{k}\right\|^{2} \\
& +2 \beta_{k}\left\|f\left(x^{k}\right)-\mu \Phi T z^{k}\right\|\left\|x^{k+1}-x^{*}\right\| \\
\leq & \left\|x^{k}-x^{*}\right\|^{2}+\alpha_{k}\left\|x^{k}-x^{k-1}\right\| 3 M-v_{k}\left(1-v_{k}\right)\left\|x^{k}-T z^{k}\right\|^{2} \\
& +2 \beta_{k}\left\|f\left(x^{k}\right)-\mu \Phi T z^{k}\right\|\left\|x^{k+1}-x^{*}\right\|,
\end{aligned}
$$

which hence yields

$$
v_{k}\left(1-v_{k}\right)\left\|x^{k}-T z^{k}\right\|^{2} \leq \Gamma_{k}-\Gamma_{k+1}+\alpha_{k}\left\|x^{k}-x^{k-1}\right\| 3 M+2 \beta_{k}\left\|f\left(x^{k}\right)-\mu \Phi T z^{k}\right\|\left\|x^{k+1}-x^{*}\right\| .
$$


Since $\beta_{k} \rightarrow 0, \Gamma_{k}-\Gamma_{k+1} \rightarrow 0, \alpha_{k}\left\|x^{k}-x^{k-1}\right\| \rightarrow 0$ and $0<\liminf _{k \rightarrow \infty} v_{k} \leq \limsup _{k \rightarrow \infty} v_{k}<1$, we infer from the boundedness of $\left\{x^{k}\right\}$ and $\left\{z^{k}\right\}$ that

$$
\lim _{k \rightarrow \infty}\left\|x^{k}-T z^{k}\right\|=0 \text {. }
$$

It follows that

$$
\begin{aligned}
\left\|x^{k+1}-x^{k}\right\| & \leq\left\|\beta_{k}\left(f\left(x^{k}\right)-\mu \Phi T z^{k}\right)+\left(1-v_{k}\right)\left(T z^{k}-x^{k}\right)\right\| \\
& \leq \beta_{k}\left\|f\left(x^{k}\right)-\mu \Phi T z^{k}\right\|+\left\|T z^{k}-x^{k}\right\| \rightarrow 0 \quad(k \rightarrow \infty) .
\end{aligned}
$$

In addition, we find

$$
\limsup _{k \rightarrow \infty}\left\langle(f-\mu \Phi) x^{*}, x_{k}-x^{*}\right\rangle \leq 0
$$

and

$$
\limsup _{k \rightarrow \infty}\left\langle(f-\mu \Phi) x^{*}, x_{k+1}-x^{*}\right\rangle \leq 0
$$

Note that $\left\{\beta_{k}(\tau-\delta)\right\} \subset[0,1], \sum_{k=1}^{\infty} \beta_{k}(\tau-\delta)=\infty$, and

$$
\limsup _{k \rightarrow \infty}\left[\frac{2\left\langle(f-\mu \Phi) x^{*}, x^{k+1}-x^{*}\right\rangle}{\tau-\delta}+\frac{3 M}{\tau-\delta} \cdot \frac{\alpha_{k}}{\beta_{k}}\left\|x^{k}-x^{k-1}\right\|\right] \leq 0 .
$$

By use of Lemma 2.2, we have $\lim _{k \rightarrow 0}\left\|x_{k}-x^{*}\right\|=0$.

Case 2. Suppose that there exits $\left\{\Gamma_{k_{l}}\right\} \subset\left\{\Gamma_{k}\right\}$ such that $\Gamma_{k_{l}}<\Gamma_{k_{l}+1}, \forall l \in \mathbf{N}$, where $\mathbf{N}$ is the set of all positive integers. Define the mapping $\tau: \mathbf{N} \rightarrow \mathbf{N}$ by

$$
\tau(k):=\max \left\{l \leq k: \Gamma_{l}<\Gamma_{l+1}\right\} .
$$

As in Case 2 of the proof of Theorem 3.1, we obtain the desired result immediately. This completes the proof.

If $F=G=0$, we derive the following result from Algorithm 3.2 and Theorem 3.2.

Corollary 3.2. Let $C$ and $Q$ be two closed convex nonempty subsets of two real Hilbert spaces $\mathscr{H}_{1}$ and $\mathscr{H}_{2}$, respectively. Let $f: C \rightarrow \mathscr{H}_{1}$ be a $\delta$-contractive mapping and $\Phi: C \rightarrow \mathscr{H}_{1}$ be $a \kappa$-Lipschitzian, $\eta$-strongly monotone mapping, where $\delta<\tau:=1-\sqrt{1-\mu\left(2 \eta-\mu \kappa^{2}\right)}$ for $\mu \in\left(0, \frac{2 \eta}{\kappa^{2}}\right)$. Let $T$ be a quasi-nonexpansive self-mapping on $C$ such that $I-T$ is demiclosed at zero and $\operatorname{Fix}(T) \cap \Gamma \neq \emptyset$, where $\Gamma=\left\{x^{*} \in C: A x^{*} \in Q\right\}$ is the solution set of the SFP. Assume $\left\{\varepsilon_{k}\right\} \subset(0,1]$ and $\left\{\beta_{k}\right\},\left\{v_{k}\right\} \subset(0,1)$ such that

(i) $\beta_{k}+v_{k} \leq 1, \sum_{k=1}^{\infty} \beta_{k}=\infty, \lim _{k \rightarrow \infty} \beta_{k}=0$;

(ii) $\varepsilon_{k}=\circ\left(\beta_{k}\right)$, i.e., $\lim _{k \rightarrow \infty} \frac{\varepsilon_{k}}{\beta_{k}}=0$;

(iii) $0<\liminf _{k \rightarrow \infty} v_{k} \leq \limsup _{k \rightarrow \infty} v_{k}<1$.

For any given $x^{0}, x^{1} \in C, \alpha>0$ and $\left\{\delta_{k}\right\} \subset[\underline{\delta}, \bar{\delta}] \subset\left(0, \frac{1}{1+\|A\|^{2}}\right)$, let the sequence $\left\{x^{k}\right\}$ be constructed as follows:

Inertial Step: Given the iterates $x^{k-1}$ and $x^{k}(k \geq 1)$, calculate $p^{k}=x^{k}+\alpha_{k}\left(x^{k}-x^{k-1}\right)$, where $\alpha_{k}$ is chosen such that $0 \leq \alpha_{k} \leq \bar{\alpha}_{k}$ and

$$
\bar{\alpha}_{k}= \begin{cases}\min \left\{\alpha, \frac{\varepsilon_{k}}{\left\|x^{k}-x^{k-1}\right\|}\right\}, & \text { if } x^{k}-x^{k-1} \neq 0 \\ \alpha, & \text { otherwise. }\end{cases}
$$


Iteration Steps: Compute $x^{k+1}$ below:

$$
\left\{\begin{array}{l}
u^{k}=P_{Q}\left(A p^{k}\right), \\
\bar{u}^{k}=P_{C}\left(p^{k}+\delta_{k} A^{*}\left(u^{k}-A p^{k}\right)\right), \\
x^{k+1}=P_{C}\left[\beta_{k} f\left(x^{k}\right)+v_{k} x^{k}+\left(\left(1-v_{k}\right) I-\beta_{k} \mu \Phi\right) T \bar{u}^{k}\right] .
\end{array}\right.
$$

Then $x^{k} \rightarrow x^{*} \in \operatorname{Fix}(T) \cap \Gamma$, which is the unique solution to the VIP: $\left\langle(\mu \Phi-f) x^{*}, p-x^{*}\right\rangle \geq 0$, $\forall p \in \operatorname{Fix}(T) \cap \Gamma$.

\section{Acknowledgements}

This paper was partially supported by the Innovation Program of Shanghai Municipal Education Commission (15ZZ068), Ph.D. Program Foundation of Ministry of Education of China (20123127110002) and Program for Outstanding Academic Leaders in Shanghai City (15XD 1503100).

\section{REFERENCES}

[1] P.K. Anh, T.V. Anh, L.D. Muu, On bilevel split pseudomonotone variational inequality problems with applications, Acta Math. Vietnam 42 (2017), 413-429.

[2] T.V. Anh, Linesearch methods for bilevel split pseudomonotone variational inequality problems, Numer. Algorithms 81 (2019), 1067-1087.

[3] E. Bonacker, A. Gibali, K.H. Küfer, Nesterov perturbations and projection methods applied to IMRT, J. Nonlinear Var. Anal. 4 (2019), 63-86.

[4] Y. Censor, A. Gibali, S. Reich, The subgradient extragradient method for solving variational inequalities in Hilbert space, J. Optim. Theory Appl. 148 (2011), 318-335.

[5] Y. Censor, A. Gibali, S. Reich, Algorithms for the split variational inequality problem, Numer. Algorithms 59 (2012), 301-323.

[6] Y. Censor, T. Elfving, A multiprojection algorithm using Bregman projections in a product space, Numer. Algorithms 8 (1994), 221-239.

[7] Y. Censor, T. Bortfeld, N. Martin, A. Trofimov, A unified approach for inversion problem in intensitymodulated radiation therapy, Phys. Med. Biol. 51 (2006), 2353-2365.

[8] Y. Censor, T. Elfving, N. Kopf, T. Bortfeld, The multiple-sets split feasiblility problem and its applications. Inverse Probl. 21 (2005) 2071-2084.

[9] L.C. Ceng, Q.H. Ansari, J.C. Yao, An extragradient method for solving split feasibility and fixed point problems, Comput. Math. Appl. 64 (2012), 633-642.

[10] S.Y. Cho, W. Li, S.M. Kang, Convergence analysis of an iterative algorithm for monotone operators, J. Inequal. Appl. 2013 (2013), Article ID 199.

[11] R.W. Cottle, J.C. Yao, Pseudo-monotone complementarity problems in Hilbert space, J. Optim. Theory Appl. 75 (2), 281-295.

[12] Q.L. Dong, Y.Y. Lu, J.F. Yang, The extragradient algorithm with inertial effects for solving the variational inequality, Optimization 65 (2016), 2217-2226.

[13] K. Goebel, S. Reich, Uniform Convexity, Hyperbolic Geometry, and Nonexpansive Mappings, Marcel Dekker, New York, 1984.

[14] G.M. Korpelevich, The extragradient method for finding saddle points and other problems, Ekonomikai Matematicheskie Metody 12 (1976), 747-756.

[15] R. Kraikaew, S. Saejung, Strong convergence of the Halpern subgradient extragradient method for solving variational inequalities in Hilbert spaces, J. Optim. Theory Appl. 163 (2014) 399-412.

[16] P.E. Maingé, Strong convergence of projected subgradient methods for nonsmooth and nonstrictly convex minimization, Set-Valued Anal. 16 (2008), 899-912. 
[17] X. Qin, J.C. Yao, Projection splitting algorithms for nonself operators, J. Nonlinear Convex Anal. 18 (2017), 925-935.

[18] X. Qin, J.C. Yao, A viscosity iterative method for a split feasibility problem, J. Nonlinear Convex Anal. 20 (2019), 1497-1506.

[19] Y. Shehu, Q.L. Dong, D. Jiang, Single projection method for pseudo-monotone variational inequality in Hilbert spaces, Optimization 68 (2019) 385-409.

[20] W. Takahashi, C.F. Wen, J.C. Yao, The shrinking projection method for a finite family of demimetric mappings with variational inequality problems in a Hilbert space, Fixed Point Theory 19 (2018), 407-419.

[21] W. Takahahsi, H.K. Xu, J.C. Yao, Iterative methods for generalized split feasibility problems in Hilbert spaces, Set-Valued Var. Anal. 23 (2015), 205-221.

[22] B. Tan, S. Xu, S. Li, Inertial shrinking projection algorithms for solving hierarchical variational inequality problems, J. Nonlinear Convex Anal. 21 (2020), 871-884.

[23] M. Tian, M. Tong, Self-adaptive subgradient extragradient method with inertial modification for solving monotone variational inequality problems and quasi-nonexpansive fixed point problems, J. Inequal. Appl. 2019 (2019), Article ID 7.

[24] D.V. Thong, D.V. Hieu, Inertial subgradient extragradient algorithms with line-search process for solving variational inequality problems and fixed point problems, Numer. Algorithms 80 (2019) 1283-1307.

[25] P.T. Vuong, Y. Shehu, Convergence of an extragradient-type method for variational inequality with applications to optimal control problems, Numer. Algorithms 81 (2019), 269-291.

[26] H.K. Xu, T.H. Kim, Convergence of hybrid steepest-descent methods for variational inequalities, J. Optim. Theory Appl. 119 (2003), 185-201.

[27] H. Zhou, X. Qin, Fixed Points of Nonlinear Operators. Iterative Methods, De Gruyter, Berlin, 2020. 